8

9

\title{
Transfer RNA-derived non-coding RNAs (tncRNAs): Uncovering hidden regulators of transcriptional regulatory circuits in plants
}

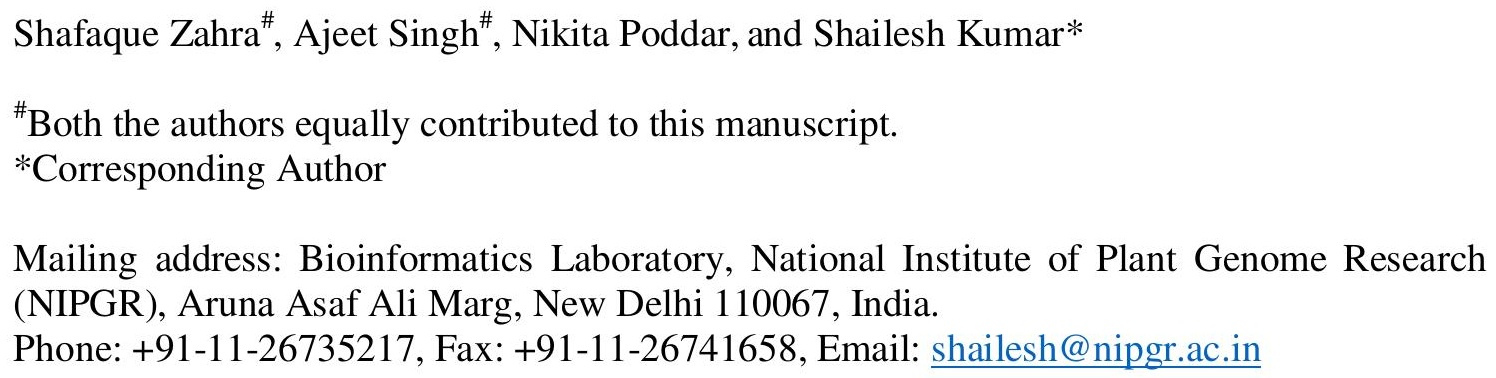

15

(6)

17

8

9

0

1

22




\section{Highlights}

- Computational pipeline for accurate identification of genuine transfer RNA-derived non-coding RNAs (tncRNAs) using small RNA sequencing (sRNA-seq) datasets.

- Six major tncRNA classes of length ranging from 14 to $50 \mathrm{nt}$ were identified in 2,500 sRNA-seq datasets in six different angiosperms.

- tRNA nucleoside modifications may affect tncRNA cleavage in plants.

- Conserved tncRNAs target transcripts are involved in plant growth, development, and metabolism.

- tncRNAs are expressed in a tissue-dependent, and stress-specific manner in plants.

\section{Abstract}

The lateral emergence of distinct classes of non-coding RNAs has led to better insights into the eukaryotic regulatory networks. Among them, the existence of transfer RNA (tRNA)derived non-coding RNAs (tncRNAs) demands their exploration in the plant kingdom. Here, we have designed a methodology to uncover the bigger picture of tncRNAome in plants. Using this, we have identified diverse tncRNAs of length 14-50 nt in 2500 small RNA sequencing (sRNA-seq) samples of six major angiosperms, and further studied their various features including length, codon-usage, cleavage pattern, and modified tRNA nucleosides. Codon-dependent generation of tncRNAs indicates that the process is highly specific rather than being mere random tRNA degradation. Analysis for nucleotide composition of tncRNA cleavage positions indicates that they are generated through precise endoribonucleolytic machinery. Certain tRNA

nucleoside modifications on tncRNAs were found to be conserved across the plants, and hence may influence tRNA cleavage, as well as tncRNA functions. Pathway enrichment analysis revealed that common tncRNA targets were majorly involved in the metabolic and developmental processes of plants. Also, many tRFs were found to be associated with transposable elements. Distinct tissue-specific tncRNA clusters indicate their role in plant development under normal physiological conditions. Furthermore, the identification of a significant number of differentially expressed tncRNAs under several abiotic and biotic stresses highlights their probable role as gene expression modulators during various stress conditions. Thus, this study will be beneficial to investigate the emerging role of tncRNAs as prospective biomarkers in plant development and stress.

\section{Keywords}

tRNA, tRFs, tRNA half, non-coding RNAs, tncRNAs, plants, computational pipeline 


\section{Introduction}

64 The profusion of non-coding RNAs (ncRNAs) with diverse regulatory actions discovered 65 over the last decade has proved to be a crucial breakthrough in the field of molecular biology.

66 They are powerful regulators of gene expression at the epigenetic, transcriptional, and post67 transcriptional levels in the living system [1]. In this diverse pool of smaller ncRNAs, 68 miRNAs and siRNAs are the most extensively surveyed molecules [2]. They alter gene 69 expression by binding to their target mRNAs [3]. The advancement of high-throughput 70 sequencing technology has tremendously resolved the surveillance of the other classes of 71 small untranslated RNAs beyond miRNAs and siRNAs [4]. Amongst them, transfer RNA 72 (tRNA)-derived non-coding RNAs (tncRNAs) have been reported in all three domains of life 73 [5]. This distinct group of regulatory RNAs is derived from the endonucleolytic cleavage of 74 precursor or mature tRNAs [6]. tncRNAs include well-known shorter tRNA-derived RNA 75 fragments up to $30 \mathrm{nt}$ lengths popularly termed as tRFs [7] (or tDRs [8]) and longer tRNA 76 halves [9] (tRHs; also recognized as tRNA-derived stress-induced fragments [10] or tiRNAs) 77 that are usually longer than $30 \mathrm{nt}$. Precise tRNA cleavage leading to their production is 78 governed by tRNA type, cell type, developmental stage, stress, and tissue [11]-[13]. It has 79 been revealed that these novel molecules can originate from both nuclear as well as 80 organellar tRNAs [14], [15]. This class of small RNAs in the range of 12-50 nucleotides (nt) 81 has been identified by various research groups and has been particularly well studied in 82 human cancers [16]-[18]. Several reports have demonstrated the tRFs as global regulators of 83 gene expression because of their association with Argonaute (AGO) proteins [19]-[21]. They 84 are frequently dysregulated in multiple types of human cancer and numerous other ailments 85 like viral infections [22], neurodegenerative [23], and metabolic disorders [24], [25]. These 86 fragments are speculated to govern diverse cellular processes such as cell proliferation [6], 87 translation initiation [26], [27], stress granule assembly [23], ribosome biogenesis [28], and 88 apoptosis [29]. In plants, the earliest report by Thompson et al in 2008 showed that the processed tRNA halves were generated in Arabidopsis subjected to oxidative stress [30]. In one work, phloemspecific tRNA fragments were shown to interfere with protein synthesis in pumpkin [31]

92 (Cucurbita maxima). In Arabidopsis, a group of 19 nt small RNAs derived from tRNAs in 93 addition to longer tRNA halves corresponding to the 5' end of tRNA, and expressed at a high 94 level in phosphate-starved roots [32]. For the first time in plants, Loss Morais and his group 
95 in 2013 showed the association of $19 \mathrm{nt}$ tRFs with Argonaute by utilizing deep sequencing 96 libraries of immunoprecipitated AGO proteins under stress conditions [33]. Alves et al. 97 extended the research and highlighted the differential accumulation of specific tRFs under abiotic stresses in Arabidopsis and rice [34]. In addition to abiotic stresses, some studies have shown links to tncRNAs generation in response to biotic stresses e.g. Apple stem grooving virus (ASGV) infected domesticated apple trees [35] and black pepper infected with Phytophthora capsici [36].

Among the eukaryotic tncRNA population, shorter tRFs range from $~ 14-30 \mathrm{nt}$ while the longer tRHs are $\sim 30-40 \mathrm{nt}$ in size [11], [37]. Depending on the position of cleavage on mature tRNA, there are two types of tRFs: 5' end derived tRF-5, and 3' end derived tRF-3 generated upon cleavage in the D and T region respectively. Among the tRNA halves, 5' or 3' tRHs are produced by cleavage in the anticodon region containing 5' or 3' portions of mature tRNA [38]. The precursor tRNA produces 5'U-tRFs/leader tRF, and tRF1/tsRNA/3'U-tRFs from the 5' leader and 3' trailer portion respectively [39]-[41]. By now, it has been established that tncRNAs can be generated in a DICER-dependent or independent manner in plants [42], [43]. Besides Dicer-like proteins (DCLs), specific endoribonucleases, RNS belonging to the RNase $\mathrm{T} 2$ family are the major contributors to the biogenesis of tncRNAs originating from mature tRNAs [34]. Both shorter and longer tncRNAs are produced by RNS1 in Arabidopsis and are evolutionarily conserved across the plant kingdom [44]. The tncRNA generation from precursor tRNAs in plants has been understudied. The nomenclature of tncRNA subcategories is based on the cleavage site of their progenitor tRNAs although a homogenized nomenclature is still lacking.

Identification of tncRNAs in small RNA-seq datasets is a very challenging and error-prone process. A major reason is the extensively modified tRNA nucleosides affect the reverse transcription process during library preparation leading to truncation or misincorporation of nucleotides [45]. This increases the probability of mismatch, thereby hampering the sequencing results. During the mapping of sequenced reads, allowing just a single mismatch to overcome sequencing errors, may also lead to base misidentification and hence causes the inflation of the false negatives. This is because of high sequence similarities among 20 major tRNA isotypes covering the whole tRNA family of isoacceptors (tRNAs with different anticodons but charged with the same amino acid), and isodecoders (tRNAs carrying the same anticodon with variations in the body sequence). Also, mapping reads to both genome 
128 increase the chance of ambiguous fragments being identified as tncRNAs. To overcome these 129 discrepancies, different mapping strategies for tRNA-derived reads have been suggested by 130 researchers [46]-[48]. Some tools are available for tncRNA detection e.g. tDRmapper [49], 131 MINTmap [50], and tRF2Cancer [51], but they are trained and tested on human datasets, and 132 thereby suit for human data only. As compared to humans, tncRNAs have been less explored 133 in planta. Although, plant exclusive databases e.g. tRex [52] and PtRFdb [53] harboring 134 information related to the transfer RNA-derived fragments (tRFs), have also been developed 135 in the recent past yet a convenient methodology for the accurate identification and 136 exploration of tncRNAs is still lacking in plants.

137 In this study, we have developed a computational methodology for the accurate identification 138 of tncRNAs in small RNA sequencing datasets and identified different kinds of tncRNAs in 139 six different Angiosperms viz. Arabidopsis thaliana (model plant), Solanum lycopersicum 140 (tomato), Cicer arietinum (chickpea), Medicago truncatula (model legume), Oryza sativa 141 (rice), and Zea mays (maize) by exploiting publicly available small RNA sequencing datasets. 142 Using our in-house built pipeline, we have identified, cataloged, and quantified tncRNAs into 143 different subtypes based on their origin and cleavage position viz. tRF-5, tRF-3, tRF-1, leader $144 \mathrm{tRF}, 5$ ' and 3' tRNA halves (tRHs). As there is no single standardized nomenclature for 145 tncRNA subtypes yet, we have followed the classical and the most frequently used terms by 146 researchers for tncRNA subtypes. tRNA modifications play an integral role in tRNA 147 maturation, structural integrity, and stabilization in all domains of life [54], [55]. These 148 modified nucleosides are also involved in a diverse range of biological activities such as gene 149 expression regulation, growth, development, and response to stresses [56], [57]. Recently, 150 few studies have indicated that some specific tRNA modifications may drive programmed 151 tRNA cleavage thus leading to the genesis of tncRNAs [58], [59]. Thus, in addition to the 152 tncRNA identification, we have also incorporated information related to the type of modified 153 nucleosides detected, and their respective position on the individual identified tncRNAs in 154 our analyzed samples. We also scrutinized tissue-specific expression of various tncRNA 155 classes from distinct tissues in Arabidopsis. It suggests the role of tissue-specific tncRNA 156 production under normal physiological conditions. Furthermore, we have also cataloged the 157 significant differentially up- and down-regulated tncRNAs under various abiotic, and biotic 158 stresses from 1000 libraries from two dicots (Arabidopsis and tomato) and two monocots 159 (rice and maize) plants. By using our methodology, this study has highlighted a major class 160 of ncRNAs derived from tRNA in plants. In addition to this, this study also provides a future 
161 perspective for better understanding the role of tRNA modifications in tRNA

162 biology. Differences in tncRNA expression in various conditions suggest their multiple role 163 in plant physiology in relevance to both normal and stress conditions.

\section{Results}

\subsection{In silico tncRNA identification across six plants}

We have identified tRNA-derived non-coding RNAs in six angiosperms by utilizing 2,660 small RNA sequencing samples available at Sequence Read Archive (SRA), from the National Center for Biotechnology Information (NCBI). We downloaded the available datasets for A. thaliana (1832), S. lycopersicum (175), C. arietinum (25), M. truncatula (139), O. sativa (258), and Z. mays (231). After processing and quality control, filtered reads were provided as input to the in-house designed pipeline for precise tncRNA identification (Fig. 1A). Due to the huge number of tRNA gene copies predicted over the entire genome by tRNAscanSE [60], we have filtered our tRNA gene pool by eliminating pseudogenes and keeping genuine tRNAs based on score. The score is an important indicator of the structural propensity of the predicted tRNA [60], [61]. Thus, only high-quality tRNAs with a score equal to or above 50 were selected for mapping. To avoid ambiguous reads coming from nontRNA regions, we created an artificial genome, by masking the genuine tRNA gene region with $50 \mathrm{nt}$ upstream and downstream in the genome and adding them as artificial chromosomes. We classified only those reads as tncRNAs that mapped exclusively to the artificial chromosomes. The identified tncRNAs were categorized into tRF-5, tRF-3, tRF-1, leader tRF, 5'tRH, and 3'tRH (Fig. 1B). Apart from them, we have also segregated the reads mapping to the internal regions of mature tRNA and collectively grouped them under other tRFs (Fig. 1B). The various information related to each tncRNA i.e. tncRNA type, parent tRNA locus information, position (start-end), sequence, length, read count, RPM, nature of modified nucleoside(s), and its position has been provided in the output file for each SRA sample. Additionally, the alignment of each tncRNA on its progenitor tRNA can be visualized in an alignment file. The count of different tncRNA subclasses for each sample is also provided in a log file.

For the analysis, we considered only those samples that generated a minimum of 100 tncRNAs, and our analyses comprise tncRNAs from 2,448 RNA-seq samples viz. A. thaliana (1676), S. lycopersicum (160), C. arietinum (21), M. truncatula (127), O. sativa (243), and Z. mays (221). The total, as well as unique tncRNAs (the same tncRNA sequences were grouped 
193 and termed as unique), counts for each plant have been summarised in Table 1. The relative

194 percentage of sRNA samples, tncRNA abundance (total and unique), tncRNA classes (total 195 and unique) for each plant are shown in Fig. 2A. The samples from Arabidopsis outnumbered 196 other plants and nearly $70 \%$ of all identified total tncRNAs belonged to the model plant. 197 Chickpea samples were the least in number, hence the tncRNAs from this plant comprised 198 only $0.01 \%$ of the total identified tncRNAs. We also observed that the relative abundance of 199 unique sequences is fairly comparable to Arabidopsis in other plants (the removal of 200 duplicate sequences coming from several samples reduces the bias of sample number). 201 Although $\sim 70 \%$ of samples were from Arabidopsis, unique tncRNAs from Arabidopsis 202 contributed to $35 \%$ of the total tncRNAs. The unique sequence count per tncRNA category 203 for individual samples from each plant has been provided in Supplementary sheet 1 (Sheets 204 1.1-1.7). The generation of tncRNAs showed a weak positive correlation (Pearson correlation 205 coefficient ranged from 0.21-0.56) with the abundance of tRNA gene copies (Supplementary 206 Figure 1). This supports the fact that the generation of tncRNAs is a non-random event and 207 the number of tncRNAs generated is not directly proportional to tRNA frequency.

\section{2.tncRNA classification according to origin and site of cleavage}

The tncRNA classes ranging from 14-50 nt were identified, and their length-wise abundance in all six plants (Fig. 2B). The tncRNAs belonging to the tRF-5 series ranged from $\sim 14-33 \mathrm{nt}$, and showed widespread distribution of varying lengths in all six plants. These fragments containing the 5' end of mature tRNA are mostly generated by cleavage in the D region. Some specifically sized fragments showed very high read counts e.g. in Arabidopsis, 24-25 nt long tRF-5s were most recurrent in the total tRF-5 population. The tncRNAs belonging to the tRF-3(CCA) series derived from the 3' end of mature tRNA by cleavage in the T region (with or without CCA at 3' end) were observed to be falling in the broad range of 14-50 nt. However, the bulk of the identified tncRNAs belonging to the tRF-3 series fall in a smaller length range up to $21 \mathrm{nt}$. The high frequency of specific-sized tncRNAs indicates cleavage preference by the endoribonuclease machinery at a certain position in the loop/stem region. Also, more than $92 \%$ of the total identified tRF-3 sequences contained terminal end CCA in all plants. The fragments corresponding to the longer halves were generated by cleavage in the anticodon region of mature tRNA. The 5' tRHs containing the terminal 5' region ranged 223 from 31-40 nt (Fig. 2B). The 3'tRH(CCA) containing the terminal 3' portion was longer than 2245 ' tRHs and ranged from 35-50 nt. Greater than 80\% of them contained CCA at the 3' end. 
226 play a role in the structural and functional integrity of fragments generated from the 3' end 227 i.e. tRF-3 series and 3' tRNA halves.

228 As compared to tncRNAs derived from mature tRNAs, the tncRNAs generated from the 229 trailer and leader sequences occupied a very tiny percentage of the total tncRNA pool. The 230 highly recurrent tRF-1 fragments fall in the range of 18-22 nt in Arabidopsis, tomato, rice, 231 and maize. Lowly expressed longer tRF-1 fragments of varying size (25-50 nt) were also 232 detected in different plants. Fragments derived from the leader region were of varying length 233 ranging from 15-50 nt. In Arabidopsis, 20 nt and longer (e.g. 48-50 nt length) fragments 234 were recurrent. Among various tncRNAs identified, greater than $40 \%$ of the reads belonged 235 to tRF-5 and tRF-3 series in individual plants (Supplementary Figure 2). We also observed a 236 remarkable fraction of fragments generated from internal portions of mature tRNA. These 237 fragments constitute nearly $45 \%$ of the total tncRNA sequences identified in each plant 238 (Supplementary Figure 2). Longer tRHs occupy less than 10\% of the tncRNAs. Compared to 239 3'tRHs, the 5' tRHs were more abundant. The remaining fraction $(<1 \%)$ comprised of 240 tncRNAs generated from pre-tRNAs.

241 Our observations confirm the existence of a heterogeneous pool of tncRNAs in plants. In the tncRNA pool, large numbers of identical tncRNAs differing only by a few base pairs were also observed in high numbers. It is still unclear how size drives the function of tncRNAs. The tncRNA length specificity may have a structure-function relationship. The length diversity of tncRNAs indicates their prospective roles besides canonical miRNA-like gene expression regulation. The high abundance of tncRNA of a specific length or falling within a narrow length range, e.g. $16 \mathrm{nt}$ tRF-5 and tRF-3 sequences need to be carefully examined as smaller fragments are usually discarded or considered to be randomly degraded fragments. The highly abundant and diverse reads belonging to tRF-5, tRF-3, as well as those derived from the internal region of tRNAs, suggest that they may have varied roles in plants whereas longer tRNA halves may be only generated during specific conditions. Although observed in low frequency than other tncRNA categories, we have observed tRF-1 expression in various samples. Till now in plants, both tRF-1 and leader tncRNAs remain unidentified because of their very low abundance and demands to be extensively explored.

\subsection{Cleavage of tncRNAs is highly specific and conserved amongst plants}

256 Nucleotide composition and the ratio of the constituent monomer units is an important 257 characteristic feature for studying nucleic acids. For studying the tncRNA cleavage pattern, 
258 we looked into the nucleotide composition of the tncRNA::tRNA junction for highly

259 recurring tRF-5, tRF-3(CCA), 5'tRH, and 3'tRH(CCA) sequences (Fig. 3). A common 260 conserved motif was observed in tRF-5::tRNA interface among all plants i.e. AG::TGG (first 261 row in Fig. 3) in tRF-5s ranging between 15-19 nt. Also, the last nucleotide in tRF-5 was 262 observed to be A/G rich (first three rows from top in Fig. 3). In tRNA::tRF-3(CCA) junction, 263 CGA(N)::(N)TC pattern was commonly observed across all species (fourth to sixth rows in 264 Fig. 3). Similarly, in the majority of the 5' tRNA halves, the breakpoint region in the middle 265 (fifth and sixth position) is either T/C (seventh to ninth rows in Fig. 3) whereas the first 266 nucleotide in $39 \mathrm{nt} 3^{\prime} \mathrm{tRH}(\mathrm{CCA})$ is A rich in all species (sixth nucleotide in the tenth rows in 267 Fig. 3). Also, a common conserved pattern, CTTGTAAAC was observed in a majority of 2683 'tRHs of 40-41nt length (eleventh and twelfth rows in Fig. 3). Despite the variability in the 269 anticodon region due to anticodon triplets in tRNA halves coming from various isoacceptors, 270 we have seen common motifs among different plants indicating that specific isoacceptors are 271 mostly preferred by the cleaving enzymes. Based on observed motifs and specific nucleotide 272 composition of tncRNAs, it is worth speculating that the enzymatic cleavage might first 273 recognize or prefer a particular motif located on parental tRNA to cleave precise fragments 274 for at least a reasonable number of genuine tncRNAs. Conservation of cleavage consensus 275 sites in different plants supports that they are the product of non-random ribonucleolytic 276 tRNA cleavage. Not only for their generation, but they may also aid in their stability and 277 interaction with their cognate binding partners. It has been reported that $\mathrm{G}$ at $18^{\text {th }}$ and $19^{\text {th }}$ 278 position in tRF-5 plays a crucial role in the inhibition of protein translation in both human 279 and Arabidopsis [62], [63]. Similarly, motif-dependent translation inhibition by 5'tRH was 280 also observed [62]. It can be speculated that at least some of the conserved residues may also 281 play a synergistic role in governing tncRNA functions.

\subsection{Specific anticodons, not the whole tRNA repertoire are responsible for tncRNA} generation

Out of the 64 triplet codons, 61 are sense codons that code for 20 amino acids. We observed that not all 61 codons generated tncRNAs. Out of 61 codons, Arabidopsis utilized 49, chickpea:41, Medicago:44, while 48 sets of tRNA isoacceptors were utilized by tomato, rice, and maize for the generation of different tncRNAs (Fig. 4). Different isoacceptors contributed to the production of different tncRNA types and varied from one plant to another. Although coding for the same amino acids, there was marked variation among the isoacceptors for 
291 isoacceptors viz. ACG, CCG, CCT, TCG, and TCT, tRNA-ArgACG generated the most

292 number of tRF-5s. Also, many anticodons may give rise to more than one class of tncRNAs 293 e.g. GluCTC, ProCGG contributed for tRF-5 and tRF-3 production in Arabidopsis. Similarly, 294 GluCTC, GlyTCC, LeuTAA generated both 5'tRHs and 3'tRHs in Arabidopsis. Also, 295 cleavage in the anticodon loop can generate both types of tRNA halves. Whether a single 296 cleavage in the tRNA molecule can generate functional 5' and 3' tRH simultaneously is 297 unclear. There must be implications pertaining to tRNA isoacceptors and the binding of cognate cleaving machinery for driving the tncRNA generation which is currently unexplored. Moreover, there can be many reasons for codon-dependent generation of tncRNAs, such as different conditions of plant growth, developmental stages, abiotic or biotic stress, etc., and also can vary from species to species.

2.5. Modification may play a major role in regulating the generation, stability, and function of tncRNAs

As tRNAs are heavily modified molecules with over 100 known modifications, we scrutinized the modifications present in our identified tncRNAs. For this, we utilized HAMR[64] which is a high utility tool for the identification of modified nucleosides in high throughput sequencing datasets. Various modifications were detected by HAMR on tncRNAs in different plants viz. 3-methylcytosine $\left(\mathrm{m}^{3} \mathrm{C}\right)$, pseudouridine $(\mathrm{Y} / \Psi)$, dihydrouridine (D), $\mathrm{N}^{2}$ methylguanosine $\left(\mathrm{m}^{2} \mathrm{G}\right), \mathrm{N}^{2}$-dimethylguanosine $\left(\mathrm{m}_{2}^{2} \mathrm{G}\right)$, 1-methyl guanosine $\left(\mathrm{m}^{1} \mathrm{G}\right), 1$ methyladenosine $\left(\mathrm{m}^{1} \mathrm{~A}\right)$, 1-methylinosine $\left(\mathrm{m}^{1} \mathrm{I}\right)$, 2-methylthio- $\mathrm{N}^{6}$-isopentenyladenosine $\left(\mathrm{ms}^{2} \mathrm{i}^{6} \mathrm{~A}\right), \mathrm{N}^{6}$-isopentenyl adenosine $\left(\mathrm{i}^{6} \mathrm{~A}\right)$, Threonylcarbamoyladenosine $\left(\mathrm{t}^{6} \mathrm{~A}\right)$ in various isoacceptors generating tncRNAs. The visualization of various modifications on tncRNAs with respect to their position on the respective consensus sequence of mature tRNA in 49 isoacceptors in Arabidopsis has been shown in Supplementary Figure 3. For other plants individually, it has been provided in Supplementary Figure 4-8. We observed that the majority of modifications were detected from $20^{\text {th }}$ to $40^{\text {th }}$ position and $50^{\text {th }}$ to $60^{\text {th }}$ position i.e. containing the cleavage sites for tRF-5, tRHs, and tRF-3, indicating a high probability of tncRNA generation to be regulated by tRNA modifications. The recurring tRNA modifications in tncRNAs from Arabidopsis samples have been highlighted in Supplementary Figure 9.

It was observed that some modifications were specific for certain anticodons and were found 
323 on GlyGCC, and iMetCAT (Supplementary Figure 9). Also pseudouridine (Y) modification

324 on GlyGCC tRNAs was highly abundant. In the anticodon loop region, Y and D were 325 abundant in tncRNAs originating from GluTTC tRNAs. In the $\mathrm{T}$ region, $\mathrm{m}^{1} \mathrm{~A}\left|\mathrm{~m}^{1} \mathrm{I}\right| \mathrm{ms}^{2} \mathrm{i}^{6} \mathrm{~A}$ 326 were observed in a large number of tRNAs. We also looked for conserved modifications on 327 different isoacceptors found common in all six plants selected for this study (Fig. 5). 328 Adenosine (A) replaced with $\mathrm{m}^{1} \mathrm{~A}\left|\mathrm{~m}^{1} \mathrm{I}\right| \mathrm{ms}^{2}{ }^{6}{ }^{6} \mathrm{~A}$ modifications were found in 20 isoacceptors viz. tRNA ArgACG|CCG|TCG|TCT ( $58^{\text {th }}$ position), AspGTC ( $57^{\text {th }}$ position), GlnTTG $\left(57^{\text {th }}\right.$ position), GlyGCC ( $57^{\text {th }}$ position), iMetCAT (57th position), LeuCAA (69 ${ }^{\text {th }}$ position), LeuCAG $\left(66^{\text {th }}\right.$ position), LeuTAG $\left(65^{\text {th }}\right.$ position), LysCTT $\left(58^{\text {th }}\right.$ position$)$, LysTTT $\left(57^{\text {th }}\right.$ position), SerAGA|CGA|TGA $\left(67^{\text {th }}\right.$ position), ThrTGT $\left(57^{\text {th }}\right.$ position), TyrGTA (58 ${ }^{\text {th }}$ position), and ValAAC|CAC (59 $9^{\text {th }}$ position). $\mathrm{G}$ is modified to $\mathrm{m}^{1} \mathrm{G}$ on the $\mathrm{T}$ loop at $58^{\text {th }}$ position in TyrGTA and $59^{\text {th }}$ position in ValAAC|CAC. These modifications were prominent in the region of the T loop which is the site of cleavage for tRF-3 generation. Also, on the $9^{\text {th }}$ nucleotide, $\mathrm{G}$ was replaced with $\mathrm{m}^{1} \mathrm{G}$ in ArgACG, GlyGCC, and iMetCAT in all plants. Various tncRNAs have been associated with abiotic stresses. E.g. Am nucleoside (2'-Omethyladenosine) is induced by salt stress in a variety of land plants including Arabidopsis, Brachypodium, poplar, and rice[65]. 2'-O-methylguanosine $(\mathrm{Gm}), 5$-methyluridine $\left(\mathrm{m}^{5} \mathrm{U}\right)$, and 5-methylcytidine $\left(\mathrm{m}^{5} \mathrm{C}\right)$ are also tightly linked to plant development [57]. The findings indicate the possibility of a very delicate cross-talk among the genetic machinery involved in tncRNA biogenesis, functioning, as well as tRNA nucleoside modification in response to plant growth, development, as well as environmental changes.

\section{6. tncRNAs are conserved amongst plants and can play a paramount role as riboregulators}

In our total analysis, we observed various tncRNAs were identical in all six plants. We found a total of 252 tRF-5, 351 tRF-3, 46 5'tRH, and 12 3'tRH common sequences. The sequence conservation provides a clue to the conservation in the regulatory function of these tncRNAs. tncRNAs can act as post-transcriptional regulators by complementary binding to their messenger RNA (mRNA) targets leading to mRNA degradation. Thus, for gaining some functional insights, some of these conserved sequences were utilized for target prediction and pathway enrichment analysis. The detailed list of conserved tRF-5s and tRF-3s along with their respective target genes have been provided in Supplementary sheet 2.1 and 2.2 respectively. We observed that several target genes were associated according to the molecular function, biological process, and cellular components for tRF-5 and tRF-3. The 
356 tRF-5 target genes were related to 9 distinct molecular functions, 25 types of biological 357 processes, and 6 cellular components (Fig. 6A). The tRF-3 target genes were associated with 35813 molecular functional categories, 26 biological activities, and 7 cellular components (Fig. $3596 \mathrm{~B})$. The target genes for the tRF-5 series were involved in various molecular functions such 360 as transferase, catalytic, kinase, endopeptidase, actin-dependent ATPase, and microfilament 361 motor activities. Genes were enriched in various metabolic processes, transport, localization, 362 and developmental processes. The target genes for the tRF-3 series were associated with 363 numerous activities like catalytic, transferase, ATPase, nucleoside-triphosphatase, RNA 364 helicase, etc. The list of all molecular functions, biological processes, and cellular 365 components along with their Gene Ontology (GO) term ID, Padj values, and $-\log _{10}\left(\right.$ Padj $_{\text {) for }}$ 366 predicted tRF-5 and tRF-3 targets is shown in Fig. 6C and Fig. 6D respectively. A detailed 367 list for tRF-5 and tRF-3 GO has been provided in Supplementary sheets 2.3 and 2.4 368 respectively. For a more organized visualization of our enrichment results, we clustered our 369 enriched genes into enrichment maps. Clusters of similar pathways representing major 370 biological processes were generated. The tRF-5 target gene clusters were enriched in growth 371 and development, transport and localization, and cellular metabolic processes were observed 372 (Fig. 6E). tRF-3 target genes formed two distinct clusters related to developmental and 373 cellular metabolic processes (Fig. 6F). It can be assumed that many tRF-5 and tRF-3 may act 374 as potent regulators of the genes involved in plant development and cellular metabolic 375 machinery in a tissue/condition-specific manner. Although many studies have shown that 376 tRFs modulate gene expression [43], [66], [67] i.e. by binding to mRNA (UTRs or exon 377 regions) in a sequence complementary manner and direct transcript cleavage, we have seen 378 that a single tRF may target multiple transcripts. Although how a single tRF can regulate 379 multiple target transcripts is still unclear to the researchers.

380 We also examined conserved tRF-5 and tRF-3 against full transposons elements (TEs) from 381 Arabidopsis. We observed that many tRF-5 and tRF-3 sequences were associated with 382 several TE transcripts (Supplementary sheets 2.5 and 2.6). A large number of tRF-5 showed 383 association with various transposon families (Fig. 7). As compared to tRF-5s, tRF-3 showed a 384 weaker association with TEs. TE belonging to the LTR/Gypsy superfamily were the most 385 frequent targets of the tRF-5 series. With this superfamily, TE from ATHILA2, ATHILA6A, 386 ATHILA4C, ATHILA4A, ATLANTYS1, ATHILA6B, and ATLANTYS2 families were found to 387 be associated with a large number of tRFs. Apart from them, TE superfamilies like 388 RC/Helitron (ATREP3 and ATREP15) and DNA/Mudr (VANDAL2 and VANDAL3) also show 
389 considerable association with tRFs. Smaller length tRFs (mostly 19-22 nt) originating from 390 AspGTC/ATC, GlnTTG, ProAGG/CGG/TGG, HisGTG, LeuCAA/TAG, and SerGCT 391 tRNAs were associated with TE elements in high numbers. Binding to TE by tncRNAs can 392 bestow genome stability to plants. Interestingly, two recent reports have shown that tRFs 393 target transposable elements (retrotransposons) in both plants and mammals, indicating their 394 potential as epigenetic regulators [68]. E.g., in Arabidopsis, a 19 nt tRF-5 from tRNA395 MetCAT cleaves its target LTR retrotransposon transcript, Athila6A [69]. Deciphering the 396 synchronization between the tncRNA generation, TE expression, and their implications in 397 plants under normal and stress conditions can unveil additional regulatory functions of this 398 class of ncRNAs.

\subsection{Identification of tissue-specific tncRNAs from Arabidopsis}

A total of 525 small RNA-seq samples (Supplementary sheet 3.1) of Arabidopsis were analyzed for the identification of tissue-specific tncRNAs. To determine whether tncRNAs show specificity towards distinct tissues in plants, we used t-Distributed Stochastic Neighbour Embedding (t-SNE), a dimensionality reduction technique to graphically simplify and explore very large datasets. We utilized this technique to explore tncRNA heterogeneity in 525 SRA samples covering 13 major tissues from the Arabidopsis plant. t-SNE plots revealed numerous tissue-specific clusters for individual tncRNA classes (Fig. 8). Seedlings and seed clusters were frequently seen in tncRNAs belonging to tRF-5, tRF-3, tRF-1, and 5'tRH. As compared to the tRNA halves, smaller fragments particularly belonging to tRF-5, and tRF-3 series showed enriched clustering for more than ten different tissues. Very few clusters were seen for 5'tRHs originating from seedlings, seed, inflorescence, silique, flower, etc. It was interesting to observe that vasculature and epidermal tissue clusters were found to be in close proximity in tRF-5, tRF-3, and 5'tRHs. Surprisingly, seedling, seed, shoot, rosette leaf, inflorescence, silique, and flower clusters were also seen in the tRF-1 series. The pattern of distribution of tncRNAs belonging to various tissues in Arabidopsis indicates that spatiotemporal expression of tncRNAs occurs in a tissue-specific manner at normal physiological conditions. Tissue-specific tncRNA signatures might regulate tissue differentiation, organ development, and regeneration in plants. 
420 To calculate the expression of tncRNAs under various abiotic and biotic stresses, a total of

421168 small RNA-seq samples viz. Arabidopsis (57), rice (36), tomato (6), and maize (69) were

422 analyzed (Supplementary sheet 3.2) We checked the expression of tncRNAs under various

423 abiotic and biotic stresses in Arabidopsis, tomato, rice, and maize (Supplementary sheet 3.3-

424 3.6). Significant differences in tncRNA expression were observed in different plants under

425 different conditions. Besides up-regulated fragments, a significant number of tncRNA were

426 down-regulated in various samples. The DE tncRNAs expressed in more than one sample

427 under different conditions in Arabidopsis, and in at least five samples in maize have been

428 shown in Fig. 9A and 9B respectively.

429 We observed stress, time, or tissue-dependent tncRNA expression in different plants. In 430 Arabidopsis, only 3 different tncRNAs showed dysregulation after $0.5 \mathrm{~h}$ of heat stress but 431 their number rose to 42 after a time duration of $6 \mathrm{~h}$. Likewise only 25 tncRNA sequences 432 were expressed drought stress at $20 \%$ field capacity (FC). However, 182 DE tncRNAs were 433 detected when FC was 30\% FC drought stress. Less than 10 DE tncRNAs were detected in 434 M. oryzae infected root tissues (9 and 7 DE tncRNAs after 30 and $120 \mathrm{~min}$ respectively) (Fig. 435 9A). However, the numbers were highly elevated in infected leaves as compared to root 436 tissues (87 and 62 tncRNAs after 30 and $120 \mathrm{~min}$ respectively). The time-dependent 437 difference in expression was also observed in few tissues in maize under heat stress (2h vs 438 48h). In RA-inoculated Arabidopsis root tissues, 212 unique tncRNA sequences were 439 differentially expressed.

440 Among all the conditions studied, we found that heat exposure leads to the maximum number 441 of differentially expressed tncRNAs in different tissues of maize, both in vegetative and 442 reproductive stages when compared with control. The highest among all with a total of 1748 443 tncRNAs were differentially expressed in tassel in reproductive stage upon exposure to 48 444 hours of heat stress, among which $35 \mathrm{nt} 5$ 'tRH derived from tRNA-GlyTCC, was 445 significantly downregulated with a $\log _{2} \mathrm{FC}$ of -30 . Of the $469 \mathrm{DE}$ tncRNAs in Arabidopsis 446 upon Alternaria brassicicola infection, 266 were upregulated and 203 were downregulated. 447 Besides, Rhizobia inoculated sample, drought $(30 \% \mathrm{FC})$ and excess $\mathrm{NaCl}$ conditions in 448 Arabidopsis revealed a considerable number of DE tncRNAs. Of the 989 DE tncRNAs that 449 were expressed in at least one stress condition in Arabidopsis, mostly ranged from 17-27nt, 450 with an abundance of $18 \mathrm{nt}$ (125) followed by $17 \mathrm{nt}$ (86), and $19 \mathrm{nt}$ (79) long tncRNAs. The 451 tomato plant when subjected to Tomato Mosaic Virus (TMV) infection showed 757 DE 452 tncRNAs, of which the majority of the fraction were contributed by $15 \mathrm{nt}$ tRFs. While the 
453 total abundance of up- and down-regulated tRFs were majorly contributed by other tRFs,

454 tRF-5 and tRF-3(CCA) were the conventionally known major tncRNAs that played an 455 important role in different stress responses. Further, certain tRFs were found to be 456 overexpressed or significantly downregulated and thus followed an unusual trend unlike the 457 tRFs from the same population; for instance, two tRF-5s (GlyTCC;27 nt and AlaAGC;24 nt 458 significantly upregulated at 30\% FC drought stress in Arabidopsis with $\log _{2}$ FC of 27.71 and 45927.62 respectively, whereas a $17 \mathrm{nt}$ tRF-5 derived from ValCAC was highly upregulated 460 upon inoculation of Alternaria brassicicola with a $\log _{2} \mathrm{FC}$ of 20.88 . Also, it was noticed that 461 DE tncRNAs in Arabidopsis were generated from HisGTG at a comparatively elevated level 462 than others, GlnCTG in maize, GluTTC in rice, and tomato.

463 We also observed DE tncRNAs belonging to the same class generating from the same tRNA. 464 As an example, two 19 mer tRF-5s from tRNA-LeuCAA (GTCAGGATGGCCGAGTGGT 465 and GTCAGGTTGGCCGAGTGGT) differing only at one nucleotide at the 7th position was 466 found to be up-regulated under $\mathrm{NaCl}$ stress. Identical tRF-5s derived from tRNA-AspGTC of $46714,16,17$, and 19 nt were all equally upregulated in RA-inoculated Arabidopsis samples. 468 This indicates that similar tncRNAs of varying length may evoke a similar response and 469 function complementarily under particular conditions. Surprisingly, some DE tRF-1 470 fragments were also detected in Arabidopsis, tomato, rice, and maize (Supplementary sheet 471 3.3-3.6). A $20 \mathrm{nt}$ tRF-1 derived from tRNA-MetCAT was accumulated under drought as well 472 as A. brassicicola infected Arabidopsis plants. In our results, we also observed the 473 upregulation of $19 \mathrm{nt}$ tRF-5 from tRNA-ArgCCT under drought (30\%FC) and $\mathrm{NaCl}$ stress in 474 Arabidopsis. The same tRF also showed elevated expression in maize leaf subjected to heat 475 stress. In earlier studies, it was reported that the same tRF was expressed at high levels in 476 Arabidopsis seedlings under drought conditions as well as in cold-treated rice inflorescences 477 [34]. The findings suggest that the accumulation of evolutionarily conserved plant tncRNAs 478 is regulated by biotic and environmental stresses. Further, validation of DE tncRNAs and 479 their putative targets will pave a way for a better understanding of the underlying mechanism in the regulation of stress responses.

482 To the best of our efforts, we have designed a workflow to accurately identify genuine 483 tncRNAs from small RNA-seq datasets. We have developed our workflow for convenient 484 tncRNA mining and can be tailored as per the requirement. Considering various 485 computational limitations in mapping tRNA fragments, we have tried to eliminate false 
486 positive and ambiguous fragments from our analysis using the computational pipeline.

487 Nevertheless, RNAs of such a small size range make it difficult to distinguish them from 488 randomly cleaved degraded products. Genome-scale study of tncRNA expression in 489 angiosperms revealed that there are various classes of tncRNAs generated from distinct 490 portions of genomic tRNAs (Fig. 10). tRNA gene clusters are localized at variable intervals 491 over the genome and they may also affect tncRNA generation. For example, tRNA-dense 492 regions can be seen particularly on Arabidopsis (chromosome 1), tomato (chromosomes 1 493 and 6), chickpea (chromosomes 1 and 5), Medicago (chromosomes 1 and 4), rice 494 (chromosomes 4 and 10), and maize (chromosomes 4 and 5). However, it can be observed 495 that the tncRNAs are not necessarily generated from tRNA-rich regions of the genome. Also, 496 all classes of tncRNAs are generated from distinct genomic regions in different plants. 497 Interestingly some regions also produce most classes of tncRNAs, like on chromosome 2 498 (Arabidopsis), chromosome 1 (tomato), chromosome 4 (Medicago and rice), and 499 chromosome 5 (maize). Some regions were producing more 5' containing tncRNAs i.e. tRF5005 and 5'tRHs, on chromosome 3 in Arabidopsis, chromosome 6 in tomato, chromosome 4 501 (Medicago), chromosome 11 (rice), and chromosome 5 (maize). It is quite conceivable that 502 some of these regions may govern rapid turn-over of tRNAs into tncRNAs. All of these 503 findings giving clues about the additional insights into the genomic organization of tRNA 504 into clusters and their implications on tRNA functions. The prevalence of tRNA gene clusters 505 in all kingdoms of life reveals various insights into evolutionary history and tRNA functions. 506 Whether tRNA gene clusters impact the generation of tncRNAs in the living system 507 including plants is worth intriguing.

508 In plants, tncRNAs can be also generated from two more organelles besides the nucleus. In 509 addition to the nucleus, the chloroplast and mitochondria also generate tncRNAs. Excluding 510 those tncRNAs that can be mapped on multiple locations, a considerable number of tncRNAs 511 originate exclusively from either nucleus, chloroplast, or mitochondria. For example, in 512 Arabidopsis, 24\% tRF-5s and 33\% tRF-3s originated from either mitochondria or chloroplast. 513 Among tRHs, 29\% 5'tRHs and 25\% 3'tRHs were exclusively derived from organellar 514 tRNAs. Overall among different plants, tncRNAs generated from plastid are more in number 515 as compared to mitochondria. However, in maize datasets, most of the organellar-derived 516 tncRNAs were mitochondrial in origin. The counts of exclusive unique tRF-5, tRF-3, 5'tRH, 517 and 3'tRH separately for all plants have been provided in Supplementary sheet 1.7. The 518 complex pool containing mitochondrial, and plastidial encoded tncRNAs detected in our 
519 datasets indicate the hidden machinery driving their biogenesis, transport in the cytosol, and

520 their functions in the cellular milieu. Although some organellar-derived tncRNAs have been 521 shown to accumulate in the cytoplasm [70], how they are generated, transported outside the 522 organelle and their functions remain to be elucidated.

523 tncRNAs particularly in the length range of 19-25 nt have been studied for the AGO 524 interaction and AGO-IP libraries have been observed to be rich in tRNA fragments [33], [34].

525 For instance, in our tissue as well as stress-related studies we have seen the abundance of 526 tncRNAs in non-AGO IP libraries as well. This also indicates that AGOs may or may not 527 associate with all tncRNAs. Apart from conventional AGO binding mediated RNA 528 interference, many tncRNAs may be functionally independent of AGO, unlike miRNAs. The 529 expression of tncRNAs in non-AGO IPs indicates some other mechanisms might also in fine530 tuning gene expression. The production of tncRNAs from precursor tRNAs has not been well 531 studied in plants. Till now in plants, tRF-1 fragments have been rarely identified and 532 characterized. In humans, tRF-1/pre-tRF-3U usually end in a short stretch of $\mathrm{T}$ residues due 533 to the release of polymerase III[6], [71]. However, in plants, most but not all tRF-1 sequences 534 have a stretch of poly(T) residues in the terminal end (Supplementary Figure 10). The 535 majority of tRF-1 sequences identified in rice, maize, and tomato samples had terminal end 536 rich in $\mathrm{T}$ residues. Interestingly, all four DE tRF-1 in tomato infected with tomato virus and 537 short DE tRF-1 (18-28 nt) in maize ended in poly(T) tail (Supplementary sheet 3.4 and 3.6). 538 Although some general mechanisms appear to be universal and conserved across kingdoms, 539 there exist additional determinants that specifically drive the biogenesis of different tRNA540 derived fragments in plants. Also, the DE tRF-1 reported in plants for the first time in our 541 study encourages us to commence the exploration of these fragments in planta.

542 The identification of genuine tncRNAs is just a head start to explore innumerable pathways 543 that are involved in the cellular machinery and to investigate their putative targets. Although 544 the in-silico approach will help in identifying these novel molecules across a wide range of 545 organisms, experimental validation, and characterization are vital for distinguishing more and 546 more bona fide tncRNAs from randomly degraded tRNA fragments. tncRNAs play biological 547 roles through a variety of mechanisms by interacting with proteins or mRNA, inhibiting 548 translation, and regulating gene expression, the cell cycle, and chromatin and epigenetic 549 modifications. Switching from conventional RNA-seq to tRNA-seq to overcome modification 550 biases will be advantageous for the identification of tncRNA. To study the molecular 551 mechanisms of tncRNAs, apart from classical molecular biology methods, such as northern 
552 blotting, microarray, real-time quantitative and reverse transcription-polymerase chain

553 reaction (qRT-PCR); advanced ribonomics [72] like cross-linking, ligation, and sequencing

554 of hybrids (CLASH); photoactivatable-ribonucleoside-enhanced cross-linking and

555 immunoprecipitation (PAR-CLIP) may lay the foundation for studying tncRNA-target hybrid

556 in plants. As tncRNAs are ubiquitous and conserved across different domains of life, our

557 comprehensive study is believed to substantiate research of these novel molecules. This will

558 aid in the investigation of non-canonical functions of tRNA, the critical role of tRNA

559 modifications in tncRNA genesis, and implications of tncRNAs in biological pathways

560 governing cellular physiology under different stress conditions, tissues, and developmental

561 stages. Their global identification will facilitate deciphering common conserved pathways in

562 eukaryotes and their mechanism of unexplored regulatory action. Leveraging computational

563 power together with molecular biology techniques will augment the current understanding of

564 the vast tncRNAomic landscape in the living system.

\section{4. Methods}

566 4.1. Data retrieval

567 Genome assembly for A. thaliana (GCF_000001735.4), S. lycopersicum (nuclear: 568 solgenomics build_4.00; organellar: GCF_000188115.4), C. arietinum (GCA_000331145.1), 569 M. truncatula (nuclear: GCF_000219495.3; organellar: NC_029641.1), O. sativa 570 (GCF_000005425.2), and Z. mays (GCF_902167145.1) were downloaded, and IDs were 571 transformed for starting with "chr", to make them convenient for secondary analysis.

572 Single-end small RNA-Seq datasets (Illumina) available for the abovementioned plants were 573 downloaded from NCBI SRA (https://www.ncbi.nlm.nih.gov/sra) using SRAToolkit (v2.10).

4.2. tRNA annotation and genome pre-processing

576 Nuclear tRNAs were predicted by tRNAscan-SE [60] (v2.0.6) using the eukaryotes model, 577 while organellar tRNAs were detected by using the '-O' option in this tool. The tRNA 578 pseudogenes and tRNAs with a score less than 50 were eliminated, and filtered tRNA regions 579 with 50 base pair (bp) flank at 3' and 5' were masked in the reference genome by 'maskfasta' 580 script of bedtools [73] (v2.29). Further, three FASTA files were prepared, 1) mature tRNA, 581 built from filtered tRNAs after intron removal, and addition of CCA at 3' terminus, 2) leader 582 tRNA, 50 bp 5' tRNA genomic region flank, and 3) trailer tRNA, 50 bp 3' tRNA genomic 583 region flank. These FASTA files were added to the masked genome as 'additional 
584 chromosomes' to create an artificial genome. The Bowtie index for the artificial genome was

585 built by bowtie-build (bowtie v1.3).

586 4.3. Identification of modification site

587 The single-end small RNA reads were processed by TrimGalore (v0.6.6) to remove the low588 quality bases, and to trim the adapter sequences. To permit error-tolerant, and uniquely 589 mapping reads alignments, processed reads were aligned to the artificial genome index using 590 bowtie[74] with '-v2 --best' options. The SAM output was sorted and converted to BAM by 591 samtools [75] (v1.10). The modification sites were predicted by HAMR [64]. Further, 592 modification sites only on mature tRNA and leader/trailer regions were only counted.

4.4. tncRNAs identification and classification

594 The mapped reads from previously generated SAM files were fetched, to create a FASTA file 595 of unique reads with the count, using SAM flag $0 \& 16$ for single-end reads. Mapped unique 596 reads were aligned to +ve strand only with no mismatch, and multi-mapped reads were 597 discarded if alignment occurred >50 times, using bowtie1 with “--norc -v 0 -m 50" 598 arguments. The bowtie combinatorial arguments "--best" and "--strata" were used which 599 guarantees that reported singleton alignments are best in terms of the stratum. Then, the 600 output was used to identify locus, location, length of read mapped to mature tRNA, and their $60150 \mathrm{bp}$ upstream and downstream flank; based on that, reads were classified into different 602 classes of tncRNAs. The tRNA halves were classified by cleavage at the anticodon loop ( $2 \mathrm{nt}$ $603+3 \mathrm{nt}$ of anticodon $+2 \mathrm{nt}=7 \mathrm{nt}$ ), and information for modification site was added for 604 tncRNAs. Reads per million (RPM) was calculated for each tncRNA by using the formula:

605 Per Million Factor $(\mathrm{PMF})=$ Total mapped reads $/ 10^{6}$

606 Reads per million $(\mathrm{RPM})=$ Number of reads mapped to progenitor tRNA / PMF

607 A local alignment file of tncRNA to the sequence of origin was also created for visualization 608 by using the pairwise 2 biopython module. The alignment score is given as per identical $=1$, non-Identical $=-1$, gap-open $=-1$, gap-extend $=-0.5$

610 4.5. tRNA model for consensus sequence and structure

611 The FASTA files were generated for specific isoacceptor tRNAs and provided as an input to 612 the LocARNA [76] (v1.9.2) software package with "--stockholm" option for consensus 613 sequence study. These 'stockholm' format files were used to create postscript files by 
614 RNAalifold [77] (v2.4.11) for the consensus tRNA sequence. The modification site(s) for 615 each tRNA isoacceptor has been shown on the consensus tRNA structures.

$616 \quad$ 4.6.Target prediction, GO, and pathway enrichment

617 The psRNATarget [78] software was utilized for tncRNA target prediction (2017 release; 618 default parameters). Common tncRNAs (tRF-5 and tRF-3 series; 18-25 nt long) were used as 619 a query while Arabidopsis cDNA and transposons sequences were used as probable targets. 620 The protein targets were analyzed for pathway enrichment analysis using g:Profiler[79] and 621 visualized in Cytoscape [80] (EnrichmentMap and AutoAnnotate).

4.7. t-Distributed Stochastic Neighbor Embedding (t-SNE) plot

t-SNE is a non-linear technique for dimensionality reduction that is particularly well suited

624 for the visualization of high-dimensional datasets. The samples sourcing from wild-type

625 Arabidopsis (Col-0) grown under normal conditions belonging to different tissues were

626 selected for this study. A total of 525 samples were utilized belonging to 13 unique tissues

627 viz. epidermis, flower, inflorescence, leaf, pollen, root, rosette leaf, seed, seedling, shoot,

628 silique, vasculature, and whole plant. A binary matrix, for presence (1) and absence (0), was

629 created for unique tncRNA sequences from each tissue sample. From this, a t-Distributed

630 Stochastic Neighbor Embedding (t-SNE) plot was generated for each aforementioned class of 631 tncRNAs. Sample and tissue information for this plot is provided in Supplementary sheet $632 \quad 3.1$.

633 4.8. Differential expression study

634 DESeq2 was utilized for the identification of differentially expressed tncRNAs under stress 635 conditions. Only those samples were selected in which at least three biological replicates for 636 each control and treatment were present (as provided in Supplementary sheet 3.2). In 637 DESeq2, the $p$-values attained by the Wald test are corrected for multiple testing using the 638 Benjamini and Hochberg method by default [81]. For our analysis, only those tncRNAs were 639 considered to be significant with $P$ less than 0.05 . Only significant tncRNAs were utilized for 640 expression analysis. tncRNAs with $\log _{2} \mathrm{FC}$ value greater than $1(>1)$, and lesser than $-1(<-1)$ 641 were considered up- and down-regulated respectively. The 'pheatmap' package of $\mathrm{R}$ was 642 used to draw the heatmaps of significant differentially expressed tncRNAs.

\section{Code and Data availability}


644 All pipeline scripts, codes, data generated, and analyzed for each of the species are available 645 at the tncRNA website (URL: http://nipgr.ac.in/tncRNA).

\section{Author contributions}

647 S.Z. and A.S. designed the pipeline, wrote the codes, and performed all the analysis. N.P. 648 contributed to the data analysis. S.Z. and SK wrote the manuscript. SK conceived the study 649 and coordinated the project.

\section{Acknowledgments}

652 S.Z. and A.S. acknowledge the Council of Scientific and Industrial Research (CSIR), India, 653 for Senior Research Fellowship. This study was supported by Grant SRG/2019/000097 from 654 Science and Engineering Research Board (SERB), Department of Science and Technology, 655 Government of India. The authors are thankful to the Department of Biotechnology (DBT)eLibrary Consortium, India, for providing access to e-resources.

\section{Funding}

658 Grant SRG/2019/000097 from Science and Engineering Research Board (SERB), 659 Department of Science and Technology, Government of India. Core research grant of 660 National Institute of Plant Genome Research, India.

\section{Conflict of interest}

662 None declared.

\section{References}

[1] P. Zhang, W. Wu, Q. Chen, and M. Chen, "Non-Coding RNAs and their Integrated Networks," Journal of integrative bioinformatics, vol. 16, no. 3. NLM (Medline), Jul. 13, 2019, doi: 10.1515/jib-2019-0027.

[2] F. F. Costa, "Non-coding RNAs: Meet thy masters," BioEssays, vol. 32, no. 7, pp. 599-608, Jul. 2010, doi: 10.1002/bies.200900112.

[3] H. Vaucheret, "Post-transcriptional small RNA pathways in plants: mechanisms and regulations.," Genes Dev., vol. 20, no. 7, pp. 759-71, Apr. 2006, doi: 10.1101/gad.1410506. 
673 [4] K. C. Vickers, L. A. Roteta, H. Hucheson-Dilks, L. Han, and Y. Guo, "Mining diverse small RNA species in the deep transcriptome," Trends Biochem. Sci., vol. 40, no. 1, pp. 4-7, Jan. 2015, doi: 10.1016/J.TIBS.2014.10.009.

[5] S. P. Keam and G. Hutvagner, "tRNA-Derived Fragments (tRFs): Emerging New Roles for an Ancient RNA in the Regulation of Gene Expression.," Life (Basel, Switzerland), vol. 5, no. 4, pp. 1638-51, Nov. 2015, doi: 10.3390/life5041638.

[6] Y. S. Lee, Y. Shibata, A. Malhotra, and A. Dutta, "A novel class of small RNAs: tRNA-derived RNA fragments (tRFs)," Genes Dev., vol. 23, no. 22, pp. 2639-2649, Nov. 2009, doi: 10.1101/gad.1837609.

[7] Y. Fu, I. Lee, Y. S. Lee, and X. Bao, "Small Non-coding Transfer RNA-Derived RNA Fragments (tRFs): Their Biogenesis, Function and Implication in Human Diseases.," Genomics Inform., vol. 13, no. 4, pp. 94-101, Dec. 2015, doi: 10.5808/GI.2015.13.4.94.

[8] R. Fricker et al., "A tRNA half modulates translation as stress response in Trypanosoma brucei," Nat. Commun., vol. 10, no. 1, pp. 1-12, Dec. 2019, doi: 10.1038/s41467-018-07949-6.

[9] J. M. Dhahbi, S. R. Spindler, H. Atamna, D. Boffelli, and D. I. K. Martin, "Deep Sequencing of Serum Small RNAs Identifies Patterns of 5' tRNA Half and YRNA Fragment Expression Associated with Breast Cancer," Biomark. Cancer, vol. 6, p. BIC.S20764, Jan. 2014, doi: 10.4137/BIC.S20764.

[10] P. Ivanov, M. M. Emara, J. Villen, S. P. Gygi, and P. Anderson, “Angiogenin-Induced tRNA Fragments Inhibit Translation Initiation,” Mol. Cell, vol. 43, no. 4, pp. 613-623, Aug. 2011, doi: 10.1016/J.MOLCEL.2011.06.022.

[11] P. Kumar, J. Anaya, S. B. Mudunuri, and A. Dutta, "Meta-analysis of tRNA derived RNA fragments reveals that they are evolutionarily conserved and associate with AGO proteins to recognize specific RNA targets," BMC Biol., vol. 12, no. 1, p. 78, Dec. 2014, doi: 10.1186/s12915-014-0078-0.

[12] M. Raina and M. Ibba, "tRNAs as regulators of biological processes," Front. Genet., vol. 5, p. 171, Jun. 2014, doi: 10.3389/fgene.2014.00171.

[13] A. R. Soares and M. Santos, "Discovery and function of transfer RNA-derived fragments and their role in disease," Wiley Interdiscip. Rev. RNA, vol. 8, no. 5, p. e1423, Sep. 2017, doi: 10.1002/wrna.1423.

[14] V. Cognat et al., "The nuclear and organellar tRNA-derived RNA fragment population in Arabidopsis thaliana is highly dynamic.," Nucleic Acids Res., vol. 45, no. 6, pp. 3460-3472, Apr. 2017, doi: 10.1093/nar/gkw1122.

[15] V. Pliatsika, P. Loher, A. G. Telonis, and I. Rigoutsos, "MINTbase: a framework for the interactive exploration of mitochondrial and nuclear tRNA fragments.," Bioinformatics, vol. 32, no. 16, pp. 2481-9, 2016, doi: 10.1093/bioinformatics/btw194.

[16] P. Anderson and P. Ivanov, "tRNA fragments in human health and disease," FEBS Lett., vol. 588, no. 23, pp. 4297-4304, Nov. 2014, doi: 10.1016/j.febslet.2014.09.001.

[17] X. Wang et al., "A Comprehensive Repertoire of Transfer RNA-Derived Fragments and Their Regulatory Networks in Colorectal Cancer," J. Comput. Biol., May 2020, doi: $10.1089 / \mathrm{cmb} .2019 .0305$. 
[18] L. Zhu, J. Ge, T. Li, Y. Shen, and J. Guo, "tRNA-derived fragments and tRNA halves: The new players in cancers," Cancer Lett., vol. 452, pp. 31-37, Jun. 2019, doi: 10.1016/J.CANLET.2019.03.012.

[19] D. Haussecker, Y. Huang, A. Lau, P. Parameswaran, A. Z. Fire, and M. A. Kay, "Human tRNA-derived small RNAs in the global regulation of RNA silencing," RNA, vol. 16, no. 4, pp. 673-695, Apr. 2010, doi: 10.1261/rna.2000810.

[20] T. Venkatesh, P. S. Suresh, and R. Tsutsumi, "tRFs: miRNAs in disguise," Gene, vol. 579, no. 2, pp. 133-138, Apr. 2016, doi: 10.1016/J.GENE.2015.12.058.

[21] M. Shigematsu and Y. Kirino, "tRNA-Derived Short Non-coding RNA as Interacting Partners of Argonaute Proteins.," Gene Regul. Syst. Bio., vol. 9, pp. 27-33, 2015, doi: 10.4137/GRSB.S29411.

[22] Q. Wang, I. Lee, J. Ren, S. S. Ajay, Y. S. Lee, and X. Bao, "Identification and Functional Characterization of tRNA-derived RNA Fragments (tRFs) in Respiratory Syncytial Virus Infection," Mol. Ther., vol. 21, no. 2, pp. 368-379, Feb. 2013, doi: 10.1038/MT.2012.237.

[23] P. Ivanov, E. O’Day, M. M. Emara, G. Wagner, J. Lieberman, and P. Anderson, “Gquadruplex structures contribute to the neuroprotective effects of angiogenin-induced tRNA fragments," Proc. Natl. Acad. Sci. U. S. A., vol. 111, no. 51, pp. 18201-18206, Dec. 2014, doi: 10.1073/pnas.1407361111.

[24] U. Sharma et al., "Biogenesis and function of tRNA fragments during sperm maturation and fertilization in mammals.," Science, vol. 351, no. 6271, pp. 391-396, Jan. 2016, doi: 10.1126/science.aad6780.

[25] Q. Chen et al., "Sperm tsRNAs contribute to intergenerational inheritance of an acquired metabolic disorder," Science (80-. )., vol. 351, no. 6271, pp. 397-400, Jan. 2016, doi: 10.1126/science.aad7977.

[26] J. Gebetsberger, L. Wyss, A. M. Mleczko, J. Reuther, and N. Polacek, "A tRNAderived fragment competes with mRNA for ribosome binding and regulates translation during stress," RNA Biol., vol. 14, no. 10, pp. 1364-1373, Oct. 2017, doi: 10.1080/15476286.2016.1257470.

[27] J. Gebetsberger, M. Zywicki, A. Künzi, and N. Polacek, "tRNA-derived fragments target the ribosome and function as regulatory non-coding RNA in Haloferax volcanii.," Archaea, vol. 2012, p. 260909, Dec. 2012, doi: 10.1155/2012/260909.

[28] H. K. Kim et al., "A transfer-RNA-derived small RNA regulates ribosome biogenesis," Nature, vol. 552, no. 7683, pp. 57-62, Nov. 2017, doi: 10.1038/nature25005.

[29] S. Blanco et al., " Aberrant methylation of t RNA s links cellular stress to neuro $\square$ developmental disorders ," EMBO J., vol. 33, no. 18, pp. 2020-2039, Sep. 2014, doi: 10.15252/embj.201489282.

[30] D. M. Thompson, C. Lu, P. J. Green, and R. Parker, "tRNA cleavage is a conserved response to oxidative stress in eukaryotes," RNA, vol. 14, no. 10, pp. 2095-2103, Aug. 2008, doi: 10.1261/rna.1232808.

[31] S. Zhang, L. Sun, and F. Kragler, "The phloem-delivered RNA pool contains small noncoding RNAs and interferes with translation.," Plant Physiol., vol. 150, no. 1, pp. 
378-87, 2009, doi: 10.1104/pp.108.134767.

[32] L.-C. Hsieh, S.-I. Lin, H.-F. Kuo, and T.-J. Chiou, "Abundance of tRNA-derived small RNAs in phosphate-starved Arabidopsis roots," Plant Signal. Behav., vol. 5, no. 5, pp. 537-539, May 2010, doi: 10.4161/psb.11029.

[33] G. Loss-Morais, P. M. Waterhouse, and R. Margis, "Description of plant tRNAderived RNA fragments (tRFs) associated with argonaute and identification of their putative targets," Biol. Direct, vol. 8, no. 1, p. 6, 2013, doi: 10.1186/1745-6150-8-6.

[34] C. S. Alves, R. Vicentini, G. T. Duarte, V. F. Pinoti, M. Vincentz, and F. T. S. Nogueira, "Genome-wide identification and characterization of tRNA-derived RNA fragments in land plants," Plant Mol. Biol., vol. 93, no. 1-2, pp. 35-48, Jan. 2017, doi: 10.1007/s11103-016-0545-9.

[35] M. Visser, H. J. Maree, D. J. G. Rees, and J. T. Burger, "High-throughput sequencing reveals small RNAs involved in ASGV infection," BMC Genomics, vol. 15, no. 1, p. 568, Jul. 2014, doi: 10.1186/1471-2164-15-568.

[36] S. Asha and E. V. Soniya, "Transfer RNA Derived Small RNAs Targeting Defense Responsive Genes Are Induced during Phytophthora capsici Infection in Black Pepper (Piper nigrum L.)," Front. Plant Sci., vol. 7, p. 767, Jun. 2016, doi: 10.3389/fpls.2016.00767.

[37] P. Kumar, C. Kuscu, and A. Dutta, "Biogenesis and Function of Transfer RNARelated Fragments (tRFs).," Trends Biochem. Sci., vol. 41, no. 8, pp. 679-689, Aug. 2016, doi: 10.1016/j.tibs.2016.05.004.

[38] J. M. Dhahbi, "5' tRNA Halves: The Next Generation of Immune Signaling Molecules.," Front. Immunol., vol. 6, p. 74, 2015, doi: 10.3389/fimmu.2015.00074.

[39] Y. S. Lee, Y. Shibata, A. Malhotra, and A. Dutta, "A novel class of small RNAs: tRNA-derived RNA fragments (tRFs)," Genes Dev., vol. 23, no. 22, pp. 2639-2649, Nov. 2009, doi: 10.1101/gad.1837609.

[40] M. Olvedy, M. Scaravilli, Y. Hoogstrate, T. Visakorpi, G. Jenster, and E. S. MartensUzunova, "A comprehensive repertoire of tRNA-derived fragments in prostate cancer," Oncotarget, vol. 7, no. 17, pp. 24766-24777, Apr. 2016, doi: 10.18632/oncotarget.8293.

[41] Y. Pekarsky et al., "Dysregulation of a family of short noncoding RNAs, tsRNAs, in human cancer.," Proc. Natl. Acad. Sci. U. S. A., vol. 113, no. 18, pp. 5071-6, May 2016, doi: 10.1073/pnas.1604266113.

[42] D. Haussecker, Y. Huang, A. Lau, P. Parameswaran, A. Z. Fire, and M. A. Kay, "Human tRNA-derived small RNAs in the global regulation of RNA silencing," RNA, vol. 16, no. 4, pp. 673-695, Apr. 2010, doi: 10.1261/rna.2000810.

[43] C. Kuscu, P. Kumar, M. Kiran, Z. Su, A. Malik, and A. Dutta, "tRNA fragments (tRFs) guide Ago to regulate gene expression post-transcriptionally in a Dicerindependent manner.," RNA, vol. 24, no. 8, pp. 1093-1105, Aug. 2018, doi: 10.1261/rna.066126.118.

[44] C. Megel et al., "Plant RNases T2, but not Dicer-like proteins, are major players of tRNA-derived fragments biogenesis," Nucleic Acids Res., vol. 47, no. 2, pp. 941-952, Jan. 2019, doi: 10.1093/nar/gky1156. 
[45] Y. Motorin, S. Muller, I. Behm-Ansmant, and C. Branlant, "Identification of Modified Residues in RNAs by Reverse Transcription-Based Methods," Methods in Enzymology, vol. 425. Academic Press, pp. 21-53, Jan. 01, 2007, doi: 10.1016/S00766879(07)25002-5.

[46] A. G. Telonis et al., "Dissecting tRNA-derived fragment complexities using personalized transcriptomes reveals novel fragment classes and unexpected dependencies," Oncotarget, vol. 6, no. 28, pp. 24797-24822, 2015, doi: 10.18632/oncotarget.4695.

[47] A. G. Telonis, P. Loher, Y. Kirino, and I. Rigoutsos, "Consequential considerations when mapping tRNA fragments.," BMC Bioinformatics, vol. 17, p. 123, Mar. 2016, doi: 10.1186/s12859-016-0921-0.

[48] A. Hoffmann, J. Fallmann, E. Vilardo, M. Mörl, P. F. Stadler, and F. Amman, "Accurate mapping of tRNA reads," Bioinformatics, vol. 34, no. 7, pp. 1116-1124, Apr. 2018, doi: 10.1093/bioinformatics/btx756.

[49] S. R. Selitsky and P. Sethupathy, "tDRmapper: challenges and solutions to mapping, naming, and quantifying tRNA-derived RNAs from human small RNA-sequencing data.," BMC Bioinformatics, vol. 16, no. 1, p. 354, Nov. 2015, doi: 10.1186/s12859015-0800-0.

[50] P. Loher, A. G. Telonis, and I. Rigoutsos, "MINTmap: fast and exhaustive profiling of nuclear and mitochondrial tRNA fragments from short RNA-seq data," Sci. Rep., vol. 7, no. 1, p. 41184, Mar. 2017, doi: 10.1038/srep41184.

[51] L.-L. Zheng et al., "tRF2Cancer: A web server to detect tRNA-derived small RNA fragments (tRFs) and their expression in multiple cancers," Nucleic Acids Res., vol. 44, no. W1, pp. W185-W193, Jul. 2016, doi: 10.1093/nar/gkw414.

[52] A. Thompson et al., "tRex: A Web Portal for Exploration of tRNA-Derived Fragments in Arabidopsis thaliana," Plant Cell Physiol., vol. 59, no. 1, pp. e1-e1, Jan. 2018, doi: $10.1093 / \mathrm{pcp} / \mathrm{pcx} 173$.

[53] N. Gupta, A. Singh, S. Zahra, and S. Kumar, "PtRFdb: a database for plant transfer RNA-derived fragments," Database, vol. 2018, no. 10, pp. 63-63, 2018, [Online]. Available: http://www.nipgr.res.in/PtRFdb/.

[54] Y. Motorin and M. Helm, "TRNA stabilization by modified nucleotides," Biochemistry, vol. 49, no. 24. American Chemical Society, pp. 4934-4944, Jun. 22, 2010, doi: 10.1021/bi100408z.

[55] B. El Yacoubi, M. Bailly, and V. De Crécy-Lagard, "Biosynthesis and function of posttranscriptional modifications of transfer RNAs," Annu. Rev. Genet., vol. 46, no. 1, pp. 69-95, Dec. 2012, doi: 10.1146/annurev-genet-110711-155641.

[56] D. Rojas-Benitez, P. C. Thiaville, V. de Crécy-Lagard, and A. Glavic, "The Levels of a Universally Conserved tRNA Modification Regulate Cell Growth.," J. Biol. Chem., vol. 290, no. 30, pp. 18699-707, Jul. 2015, doi: 10.1074/jbc.M115.665406.

[57] Y. Wang et al., "Identification of tRNA nucleoside modification genes critical for stress response and development in rice and Arabidopsis," BMC Plant Biol., vol. 17, no. 1, p. 261, Dec. 2017, doi: 10.1186/s12870-017-1206-0.

[58] S. M. Lyons, M. M. Fay, and P. Ivanov, "The role of RNA modifications in the 
regulation of tRNA cleavage," FEBS Lett., vol. 592, no. 17, pp. 2828-2844, Sep. 2018, doi: 10.1002/1873-3468.13205.

[59] M. Pereira, D. R. Ribeiro, M. M. Pinheiro, M. Ferreira, S. Kellner, and A. R. Soares, "m 5 U54 tRNA hypomodification by lack of TRMT2A drives the generation of tRNA-derived small RNAs," no. January, 2021, doi: 10.20944/preprints202101.0227.v1.

[60] T. M. Lowe and S. R. Eddy, "TRNAscan-SE: A program for improved detection of transfer RNA genes in genomic sequence," Nucleic Acids Res., vol. 25, no. 5, pp. 955964, 1996, doi: 10.1093/nar/25.5.0955.

[61] T. Pan, "Modifications and functional genomics of human transfer RNA," Cell Res., vol. 28, no. 4, pp. 395-404, Apr. 2018, doi: 10.1038/s41422-018-0013-y.

[62] A. Sobala and G. Hutvagner, "Small RNAs derived from the 5' end of tRNA can inhibit protein translation in human cells.," RNA Biol., vol. 10, no. 4, pp. 553-63, Apr. 2013, doi: 10.4161/rna.24285.

[63] S. Lalande, R. Merret, T. Salinas-Giegé, and L. Drouard, "Arabidopsis tRNA-derived fragments as potential modulators of translation," RNA Biol., vol. 17, no. 8, pp. 11371148, Aug. 2020, doi: 10.1080/15476286.2020.1722514.

[64] P. Ryvkin et al., "HAMR: High-throughput annotation of modified ribonucleotides," $R N A$, vol. 19, no. 12, pp. 1684-1692, Dec. 2013, doi: 10.1261/rna.036806.112.

[65] Y. Wang et al., "The 2'-O-methyladenosine nucleoside modification gene OsTRM13 positively regulates salt stress tolerance in rice," J. Exp. Bot., vol. 68, no. 7, pp. 14791491, Mar. 2017, doi: 10.1093/jxb/erx061.

[66] H. Goodarzi, X. Liu, H. C. B. Nguyen, S. Zhang, L. Fish, and S. F. Tavazoie, "Endogenous tRNA-Derived Fragments Suppress Breast Cancer Progression via YBX1 Displacement," Cell, vol. 161, no. 4, pp. 790-802, May 2015, doi: 10.1016/J.CELL.2015.02.053.

[67] S. Karaiskos, A. S. Naqvi, K. E. Swanson, and A. Grigoriev, "Age-driven modulation of tRNA-derived fragments in Drosophila and their potential targets," Biol. Direct, vol. 10, no. 1, p. 51, Dec. 2015, doi: 10.1186/s13062-015-0081-6.

[68] G. Martinez, "tRNA-derived small RNAs: New players in genome protection against retrotransposons," RNA Biol., vol. 15, no. 2, pp. 170-175, Feb. 2018, doi: 10.1080/15476286.2017.1403000.

[69] G. Martinez, S. G. Choudury, and R. K. Slotkin, "tRNA-derived small RNAs target transposable element transcripts," Nucleic Acids Res., vol. 45, no. 9, pp. 5142-5152, May 2017, doi: 10.1093/nar/gkx103.

[70] V. Cognat et al., "The nuclear and organellar tRNA-derived RNA fragment population in Arabidopsis thaliana is highly dynamic," Nucleic Acids Res., vol. 45, no. 6, pp. 3460-3472, Apr. 2017, doi: 10.1093/nar/gkw1122.

[71] V. Balatti et al., "tsRNA signatures in cancer," Proc. Natl. Acad. Sci. U. S. A., vol. 114, no. 30, pp. 8071-8076, Jul. 2017, doi: 10.1073/pnas.1706908114.

[72] M. Dziublenski, A. N. Roff, and F. T. Ishmael, "Ribonomic approaches to identify protein-mRNA and microRNA-mRNA interactions: Implications for drug design," 
Drug Dev. Res., vol. 73, no. 7, pp. 406-413, 2012, doi: 10.1002/ddr.21031.

[73] A. R. Quinlan and I. M. Hall, "BEDTools: a flexible suite of utilities for comparing genomic features," Bioinformatics, vol. 26, no. 6, pp. 841-842, Mar. 2010, doi: 10.1093/bioinformatics/btq033.

[74] B. Langmead, C. Trapnell, M. Pop, and S. L. Salzberg, "Ultrafast and memoryefficient alignment of short DNA sequences to the human genome," Genome Biol., vol. 10, no. 3, p. R25, Mar. 2009, doi: 10.1186/gb-2009-10-3-r25.

[75] H. Li et al., "The Sequence Alignment/Map format and SAMtools.," Bioinformatics, vol. 25, no. 16, pp. 2078-9, Aug. 2009, doi: 10.1093/bioinformatics/btp352.

[76] S. Will, T. Joshi, I. L. Hofacker, P. F. Stadler, and R. Backofen, "LocARNA-P: Accurate boundary prediction and improved detection of structural RNAs," RNA, vol. 18, no. 5, pp. 900-914, May 2012, doi: 10.1261/rna.029041.111.

[77] S. H. Bernhart, I. L. Hofacker, S. Will, A. R. Gruber, and P. F. Stadler, "RNAalifold: improved consensus structure prediction for RNA alignments," BMC Bioinformatics, vol. 9, no. 1, p. 474, Dec. 2008, doi: 10.1186/1471-2105-9-474.

[78] X. Dai, Z. Zhuang, and P. X. Zhao, "PsRNATarget: A plant small RNA target analysis server (2017 release)," Nucleic Acids Res., vol. 46, no. W1, pp. W49-W54, Jul. 2018, doi: 10.1093/nar/gky316.

[79] U. Raudvere et al., "g:Profiler: a web server for functional enrichment analysis and conversions of gene lists (2019 update)," Nucleic Acids Res., no. 1, 2019, doi: 10.1093/nar/gkz369.

[80] P. Shannon et al., "Cytoscape: A software Environment for integrated models of biomolecular interaction networks," Genome Res., vol. 13, no. 11, pp. 2498-2504, Nov. 2003, doi: 10.1101/gr.1239303.

[81] M. I. Love, W. Huber, and S. Anders, "Moderated estimation of fold change and dispersion for RNA-seq data with DESeq2," Genome Biol., vol. 15, no. 12, p. 550, Dec. 2014, doi: 10.1186/s13059-014-0550-8. 


\section{Table}

936 Table 1: Summary of identified transfer-RNA-derived non-coding RNAs (tncRNAs) 937 abundance in six plants.

\begin{tabular}{|c|c|c|c|c|c|c|c|c|c|}
\hline & & tncRNAs & tRF-5 & tRF-3(CCA) & tRF-1 & $5^{\prime} \mathrm{tRH}$ & $3^{\prime} \mathrm{tRH}(\mathrm{CCA})$ & Leader tRF & Other tRFs \\
\hline $\begin{array}{c}\text { Plant } \\
\text { species }\end{array}$ & $\begin{array}{c}\text { Total } \\
\text { Samples }\end{array}$ & $\begin{array}{c}\text { Total } \\
\text { /Unique }\end{array}$ & $\begin{array}{c}\text { Total } \\
\text { /Unique }\end{array}$ & $\begin{array}{c}\text { Total } \\
\text { /Unique }\end{array}$ & $\begin{array}{c}\text { Total } \\
\text { /Unique }\end{array}$ & $\begin{array}{c}\text { Total } \\
\text { /Unique }\end{array}$ & $\begin{array}{l}\text { Total } \\
\text { /Unique }\end{array}$ & $\begin{array}{c}\text { Total } \\
\text { /Unique }\end{array}$ & $\begin{array}{l}\text { Total } \\
\text { /Unique }\end{array}$ \\
\hline A. thaliana & 1676 & $\begin{array}{c}3362128 \\
/ 50619\end{array}$ & $\begin{array}{l}760155 \\
/ 2180\end{array}$ & $\begin{array}{c}782722 \\
/ 4513\end{array}$ & $\begin{array}{c}22505 \\
/ 693\end{array}$ & $\begin{array}{c}164074 \\
1662\end{array}$ & $\begin{array}{c}28835 \\
/ 840\end{array}$ & $\begin{array}{l}2359 \\
/ 238\end{array}$ & $\begin{array}{l}1601478 \\
/ 41502\end{array}$ \\
\hline C. arietinum & 21 & $\begin{array}{l}54390 \\
/ 10627\end{array}$ & $\begin{array}{l}8837 \\
/ 1012\end{array}$ & $\begin{array}{l}11763 \\
/ 1792\end{array}$ & $\begin{array}{l}77 \\
/ 35\end{array}$ & $\begin{array}{l}2135 \\
/ 306\end{array}$ & $\begin{array}{l}778 \\
/ 175\end{array}$ & $\begin{array}{l}26 \\
/ 12\end{array}$ & $\begin{array}{l}30774 \\
17310\end{array}$ \\
\hline M. truncatula & 127 & $\begin{array}{l}306836 \\
/ 11971\end{array}$ & $\begin{array}{l}63157 \\
/ 1182\end{array}$ & $\begin{array}{l}82262 \\
/ 1967\end{array}$ & $\begin{array}{l}779 \\
172 \\
\end{array}$ & $\begin{array}{c}15232 \\
/ 321\end{array}$ & $\begin{array}{l}4372 \\
/ 139\end{array}$ & $\begin{array}{l}137 \\
132\end{array}$ & $\begin{array}{l}140897 \\
/ 8270\end{array}$ \\
\hline O. sativa & 243 & $\begin{array}{l}406496 \\
/ 23117\end{array}$ & $\begin{array}{c}100828 \\
/ 1812\end{array}$ & $\begin{array}{l}98089 \\
/ 2787\end{array}$ & $\begin{array}{l}1553 \\
/ 189\end{array}$ & $\begin{array}{c}19070 \\
1447\end{array}$ & $\begin{array}{l}2595 \\
/ 294\end{array}$ & $\begin{array}{l}53 \\
/ 26\end{array}$ & $\begin{array}{l}184308 \\
/ 17585\end{array}$ \\
\hline $\begin{array}{l}\text { S. } \\
\text { lycopersicum }\end{array}$ & 160 & $\begin{array}{l}265435 \\
/ 23312\end{array}$ & $\begin{array}{l}62838 \\
/ 1753\end{array}$ & $\begin{array}{l}59833 \\
/ 3323\end{array}$ & $\begin{array}{l}1878 \\
/ 688\end{array}$ & $\begin{array}{c}19298 \\
/ 560\end{array}$ & $\begin{array}{l}2429 \\
/ 264\end{array}$ & $\begin{array}{l}130 \\
178\end{array}$ & $\begin{array}{l}119029 \\
/ 16664\end{array}$ \\
\hline Z. mays & 221 & $\begin{array}{l}428193 \\
/ 18304\end{array}$ & $\begin{array}{l}95748 \\
/ 1819\end{array}$ & $\begin{array}{l}99510 \\
/ 2651\end{array}$ & $\begin{array}{l}1959 \\
/ 265\end{array}$ & $\begin{array}{c}37768 \\
/ 618\end{array}$ & $\begin{array}{l}7457 \\
/ 354\end{array}$ & $\begin{array}{l}164 \\
/ 27\end{array}$ & $\begin{array}{l}185587 \\
/ 12584\end{array}$ \\
\hline
\end{tabular}

\section{$939 \quad$ Figures}

\section{Figure 1: Identification of transfer-RNA-derived non-coding RNAs (tncRNAs). A}

941 Workflow for the identification of various classes of tncRNAs using reference genome, and

942 processed small RNA-seq data based on tRNA origin, and site of cleavage. B Biogenesis of

943 distinct tncRNA subtypes generated from the excision of precursor, and mature tRNA. pre-

944 tRNA gives rise to leader tRF and tRF-1/tsRNA, whereas mature tRNA generates tRF-5,

945 tRF-3(CCA), 5'tRH, 3'tRH(CCA), and other tRFs (derived from internal portions excluding

946 extreme ends of mature tRNA denoted by dashed lines). 
947 Figure 2: Abundance of the identified transfer-RNA-derived non-coding RNAs

948 (tncRNAs) in different plants. A Relative distribution of samples, total and unique tncRNA, 949 tRF-5, tRF-3(CCA), tRF-1, 5'tRH, 3'tRH(CCA), leader, and other tRFs analyzed in this 950 study. B Size distribution of major tncRNA categories viz. tRF-5, tRF-3(CCA), tRF-1, 9515 'tRH, 3'tRH(CCA), and other tRFs in individual plants.

952 Figure 3: Web logo representation depicting conserved cleavage sites for four tncRNA 953 subclasses viz. tRF-5, tRF-3(CCA), 5'tRH, and 3'tRH(CCA). Web Logos were made 954 using top-three recurring tncRNAs by taking $5 \mathrm{nt}$ stretches from progenitor tRNA and 955 respective tncRNA fragments viz. tRF-5::tRNA; 5'tRH::tRNA; tRNA::tRF956 3(CCA);tRNA::3'tRH(CCA). To study the breakpoint and its flanking nucleotide 957 composition, for tRF-5 and 5'tRH classes, the first five nucleotides (from left to right) 958 represent the last five nucleotides of tRF-5/5'tRH and the remaining stretch of nucleotides is 959 the tRNA (sixth to the eleventh nucleotide). For tRF-3(CCA) and 3'tRH(CCA) classes, the 960 first five nucleotides (from left to right) represent progenitor tRNA sequence, and the 961 remaining stretch represents the first sixth nucleotide of tRF-3(CCA)/3'tRH(CCA) (sixth to 962 the eleventh nucleotide). The red arrow represents the breaking point of each tncRNA 963 fragment from its parental tRNA. All the sequences shown are oriented in the 5' to 3' 964 direction. G|T; T|C; A|A breakpoint was commonly found in all types of tncRNAs.

Figure 4: Heatmap showing the abundance of tncRNA subclasses viz. tRF-5, tRF-3(CCA), tRF-1, 5'tRH, 3'tRH(CCA), and leader tRF sourcing from each tRNA isoacceptor (specified using their respective anticodon) in six plants; The frequencies are given in read count converted to $\log 10$ values.

Figure 5: tRNA isoacceptor secondary structure models showing common nucleoside modifications in all six plants. Circles (in yellow) depict the position of modification residue on the tncRNA generated from the respective tRNA.

Figure 6: Pathway Enrichment analysis for conserved tRF-5 and tRF-3 target genes.

973 A,B Manhattan plot illustrating the enriched terms across the analyzed GO term categories 974 for tRF-5 and tRF-3 targets respectively. The x-axis shows the functional terms and the 975 corresponding enrichment P-values in the negative $\log 10$ scale are represented on the $y$-axis. 976 Each color-coded circle on the plot represents a single functional term. C,D Detailed results 977 in tabular output illustrating GO term name, term ID, and P-values (in order of the most to 978 least significant) for tRF-5 and tRF-3 targets respectively. E,F Enrichment map visualization 
979 of enriched pathways generated using. G:Profiler analysis for tRF-5 and tRF-3 target genes respectively. Pathways are shown as circles (nodes) that are connected with lines (edges) if the pathways share multiple genes. Nodes and edges were manually formatted to present a neat picture.

Figure 7: A chord diagram representing the connections between conserved tRF-5s and their corresponding target transposable elements (TE) family and their superfamily. Most recurring TE family and associated tRF-5 have been labelled. Broader fragment reflects the greater number of associations.

Figure 8: t-Distributed Stochastic Neighbor Embedding (t-SNE) for the visualization of the tissue-specific tncRNA expression in A. thaliana (wild type under normal conditions) datasets. Individual t-SNE plot was made for tRF-5, tRF-3, tRF-1, 5'tRH, and 3'tRH classes. The tncRNAs from a total of 525 samples from 13 different tissues were utilized for this analysis. Tissue clusters observed have been shown by similar color-coded dashed circles. tncRNAs in A. thaliana in eleven different types of stress from left to right viz. heat stress (0.5hr), heat stress (6hr), drought stress (at 20\% field capacity), drought stress (at $30 \%$ field capacity), sulfur deficiency, cadmium excess, copper deficiency, copper excess, $\mathrm{NaCl}$ excess, Alternaria brassicicola infection, and Rhizobacteria inoculated samples. B DE expressed tncRNAs in Z. mays in fifteen different conditions with respect to stress condition and tissue origin viz. drought, salt, drought plus salt, heat stress $(2 \mathrm{hr}, 48 \mathrm{hr})$ in various reproductive and vegetative tissues. To reduce the large number of DE tncRNAs detected in various samples, we have represented only those tncRNAs in the heatmap occurring in at least 2 samples in Arabidopsis and five or more samples in maize. The full list DE tncRNAs for Arabidopsis and maize has been provided in Supplementary sheet 3.3 and 3.6 respectively. 
1011 seventh ring shows the genomic tRNA region producing tRF-5, tRF-3(CCA), tRF-1, 5' tRH, 1012 and 3'tRH respectively. The innermost ring shows the tRNA gene distribution across the 1013 genome. tncRNA/tRNA-rich regions shown in purple (highest density) and tncRNA/tRNA1014 poor regions shown in the red (minimum density) spectrum. 


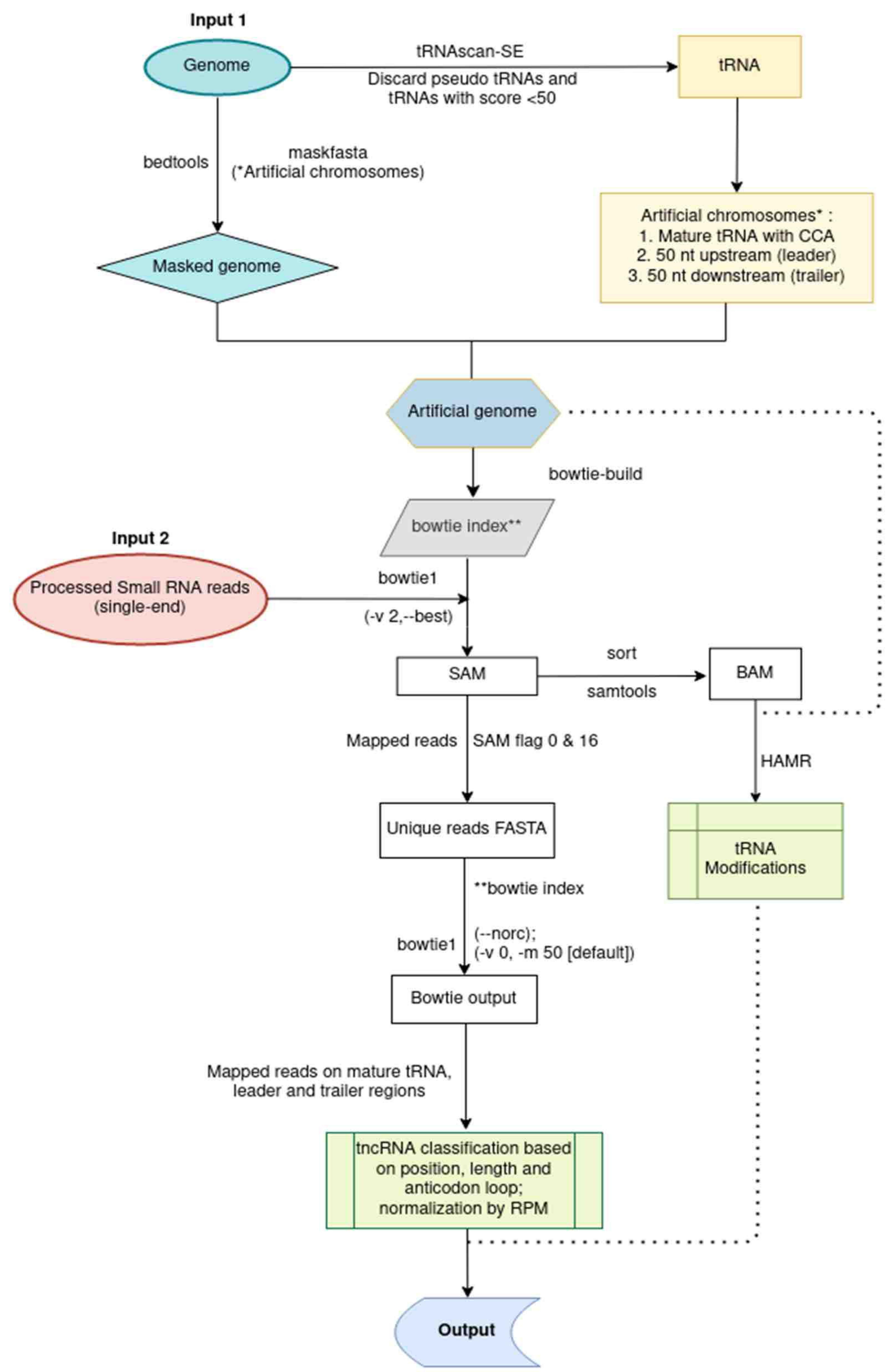

B

Leader region

Trailer region

$\mathbf{5}^{\prime}$

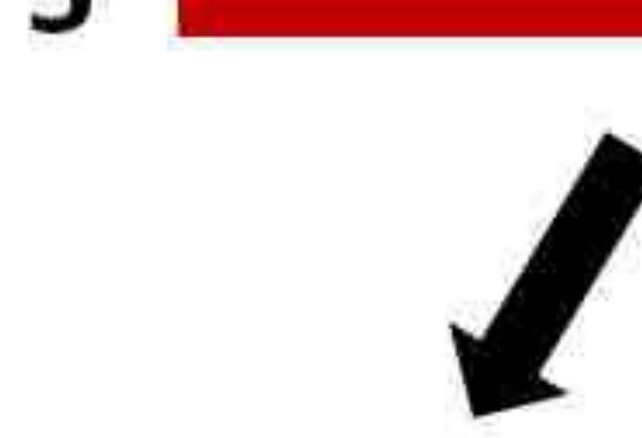

Leader tRF

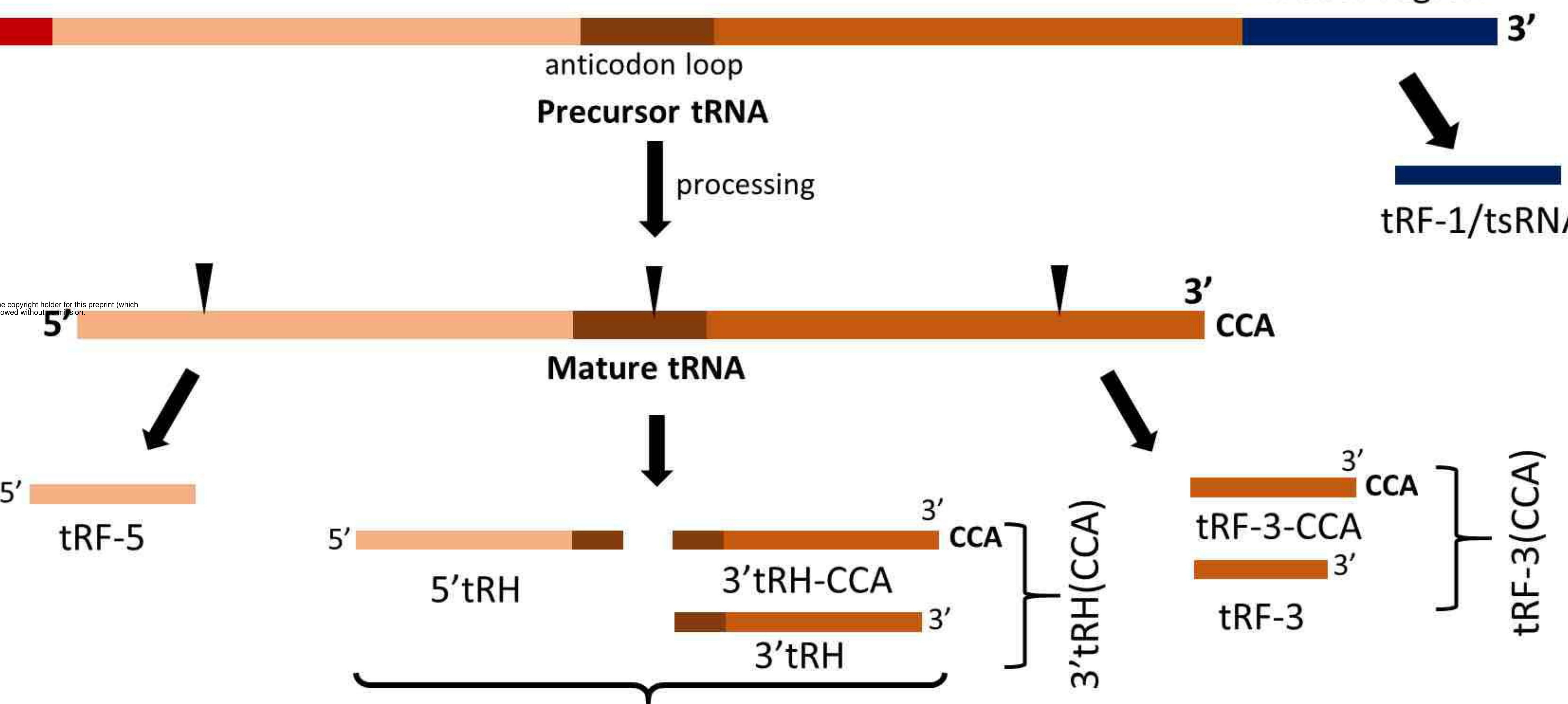

tRNA halves

L.

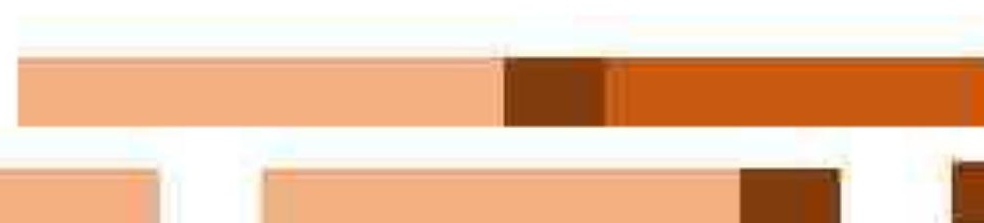





M. truncatula

O. sativa

Z. mays

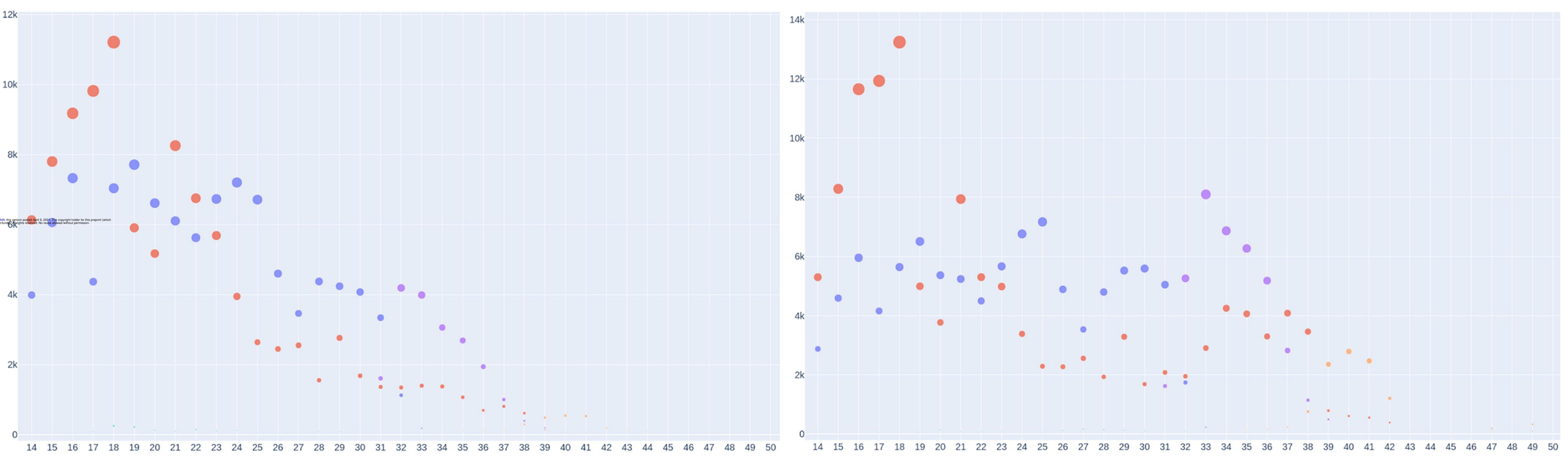



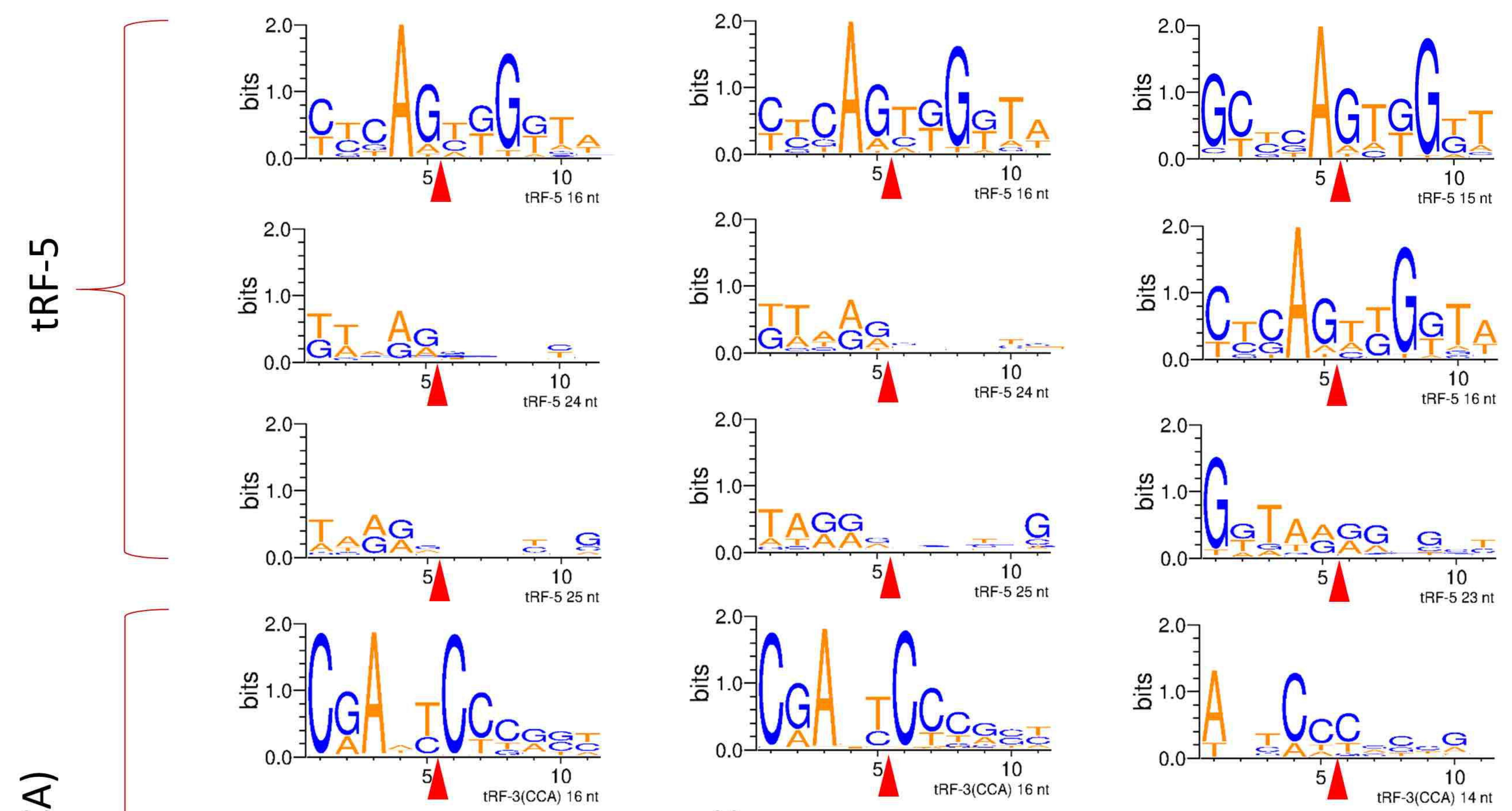

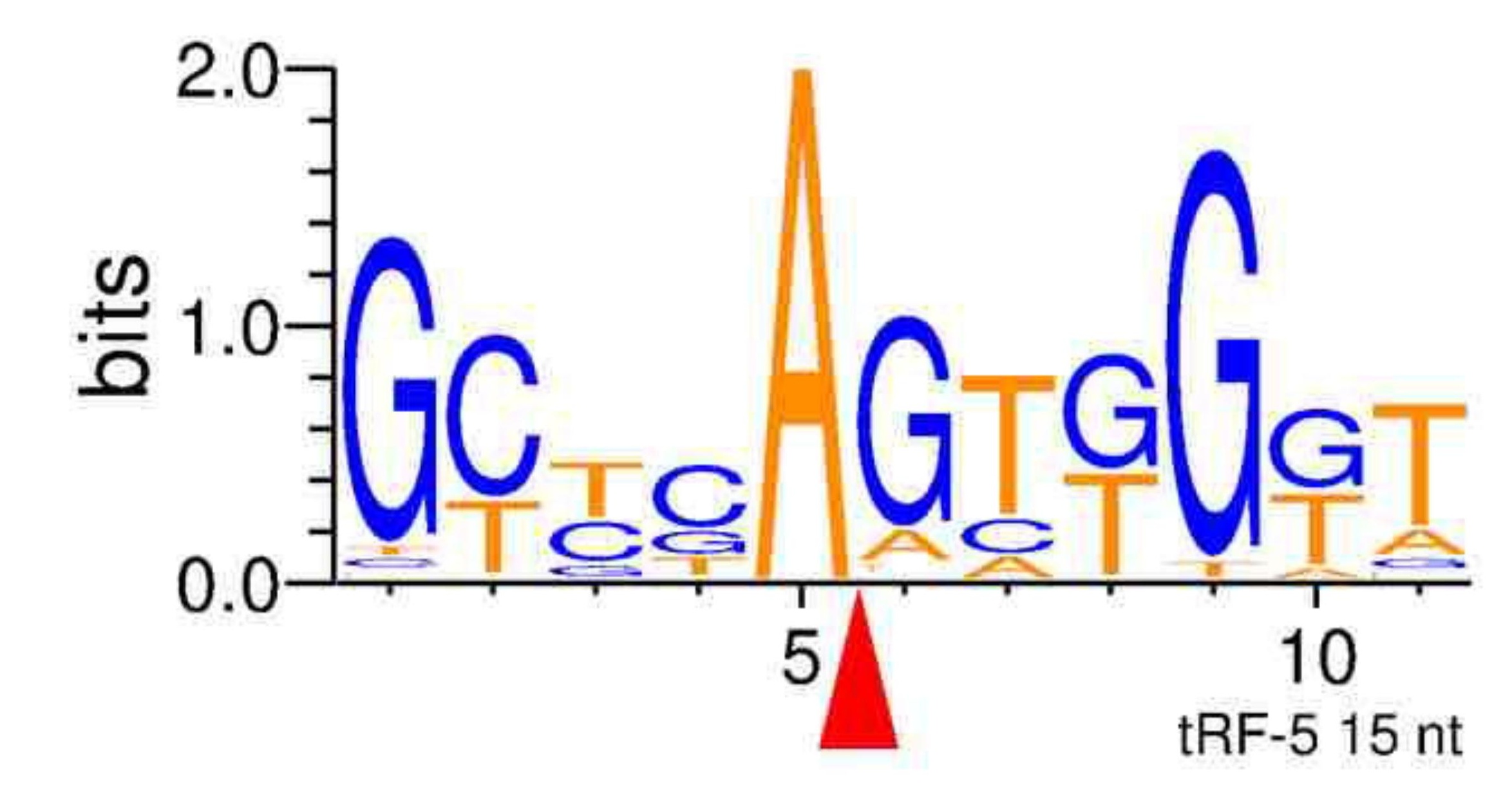
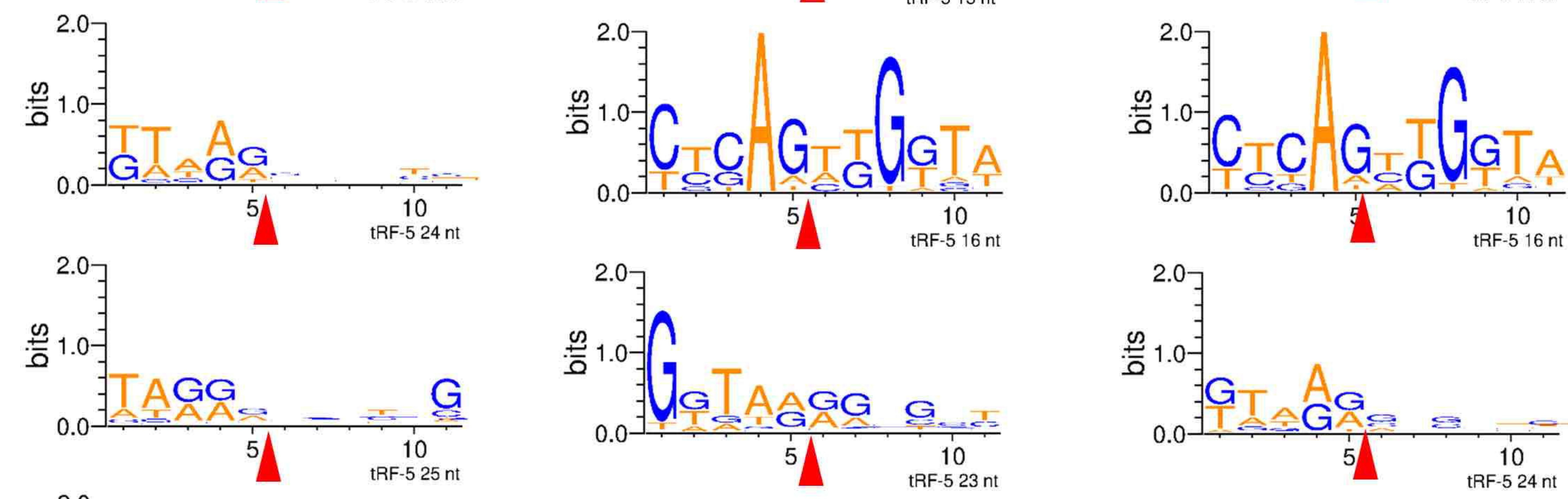

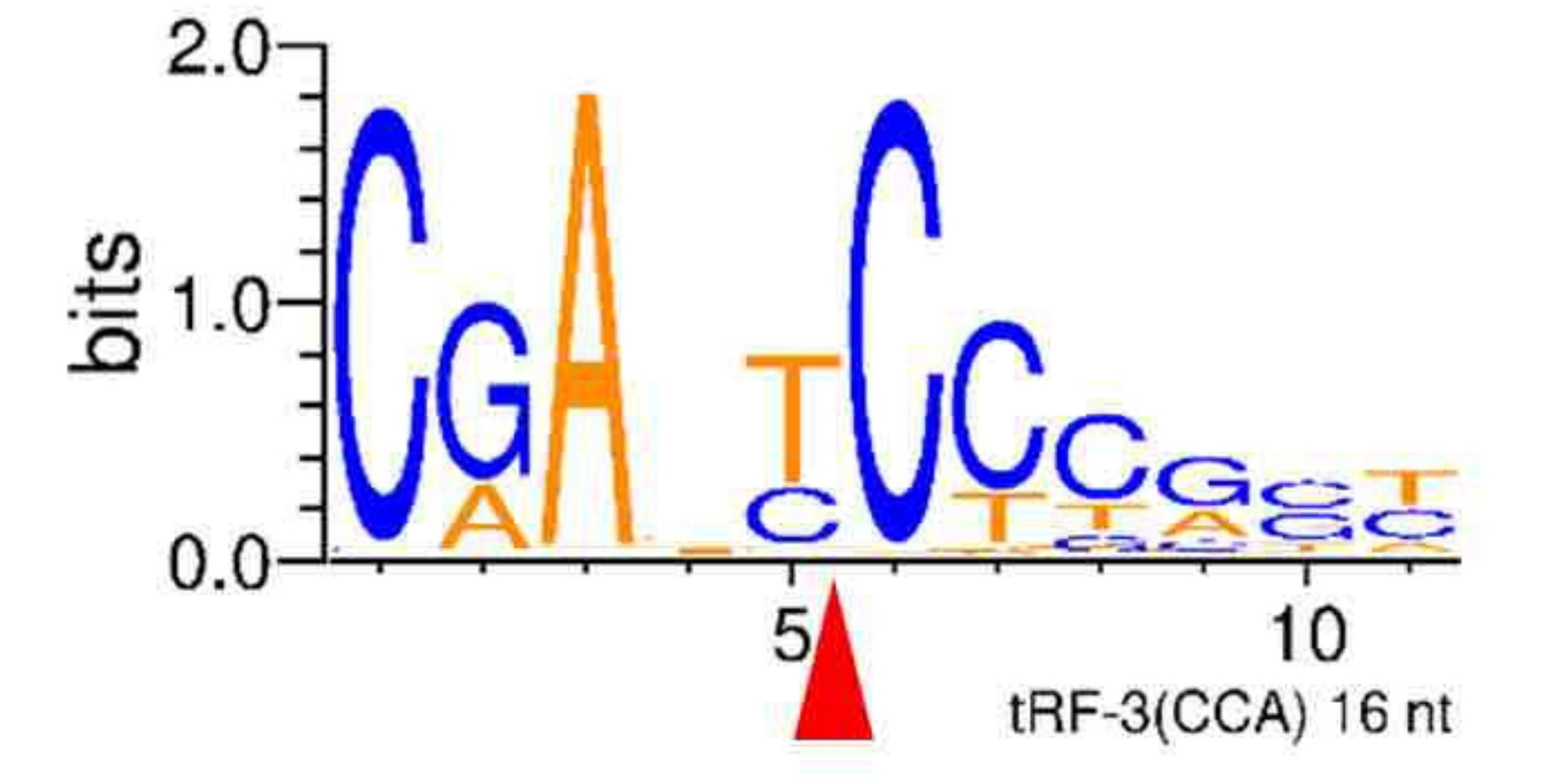
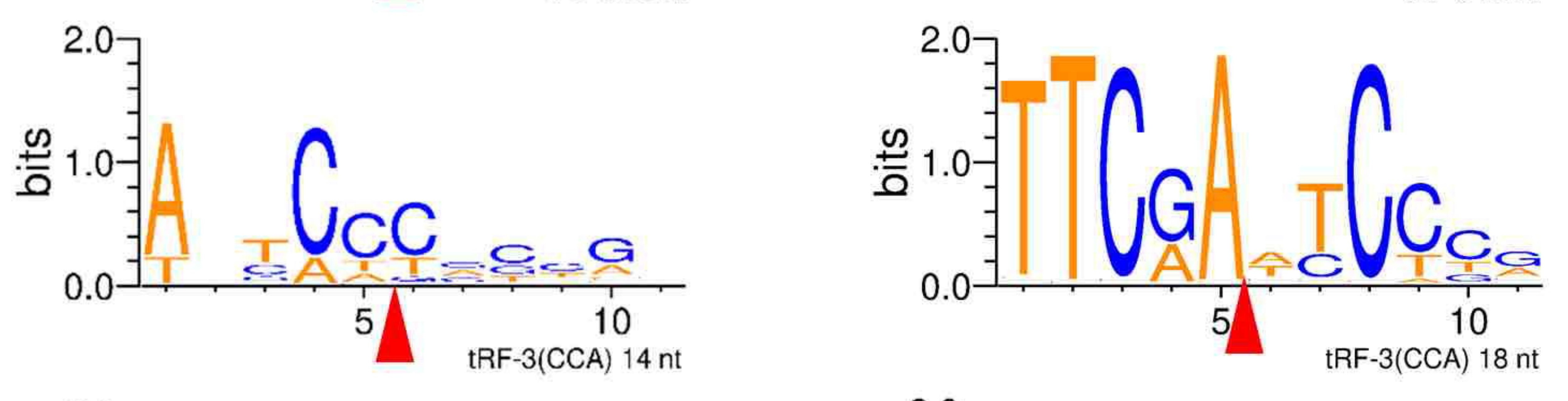

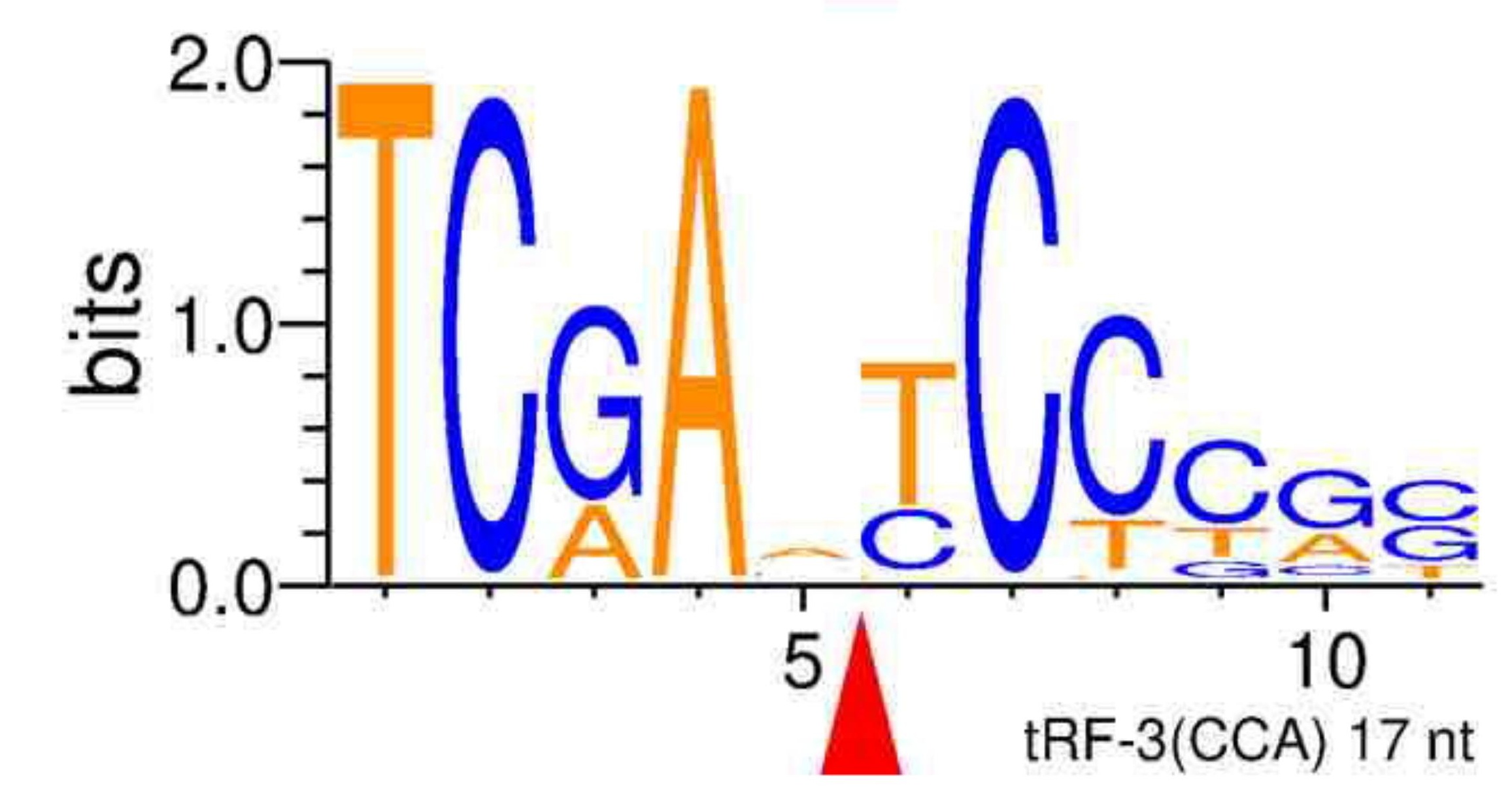

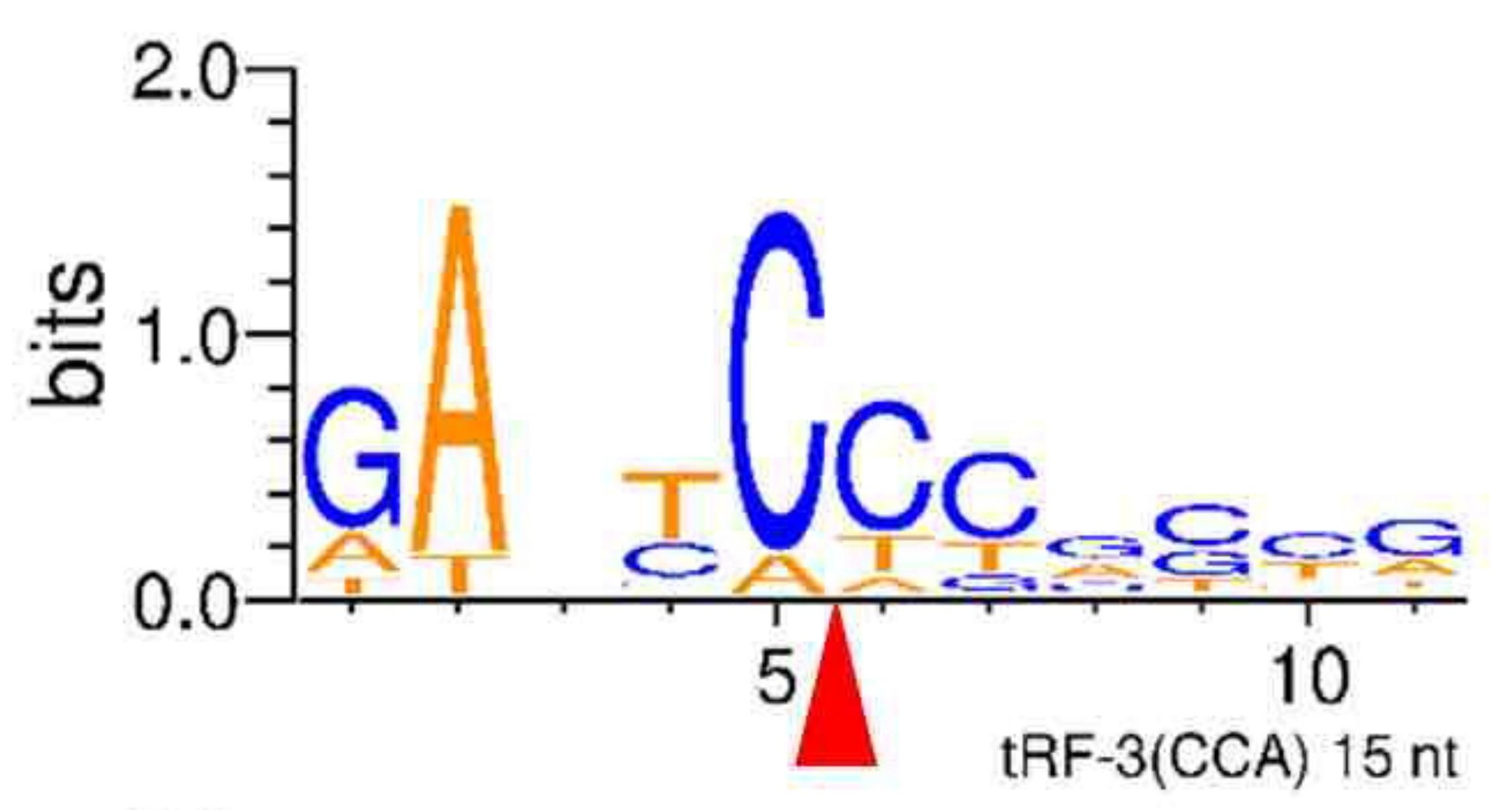

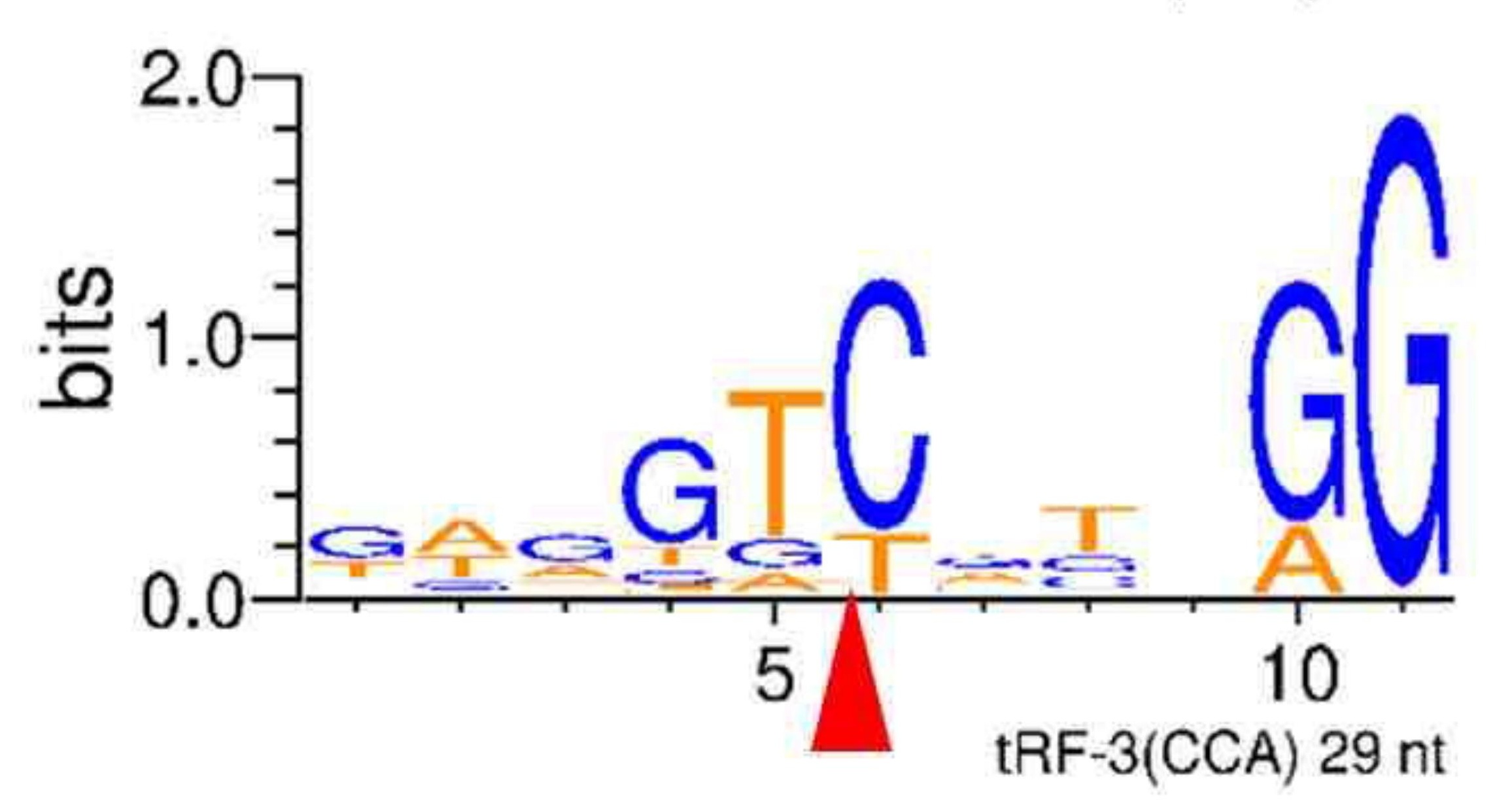

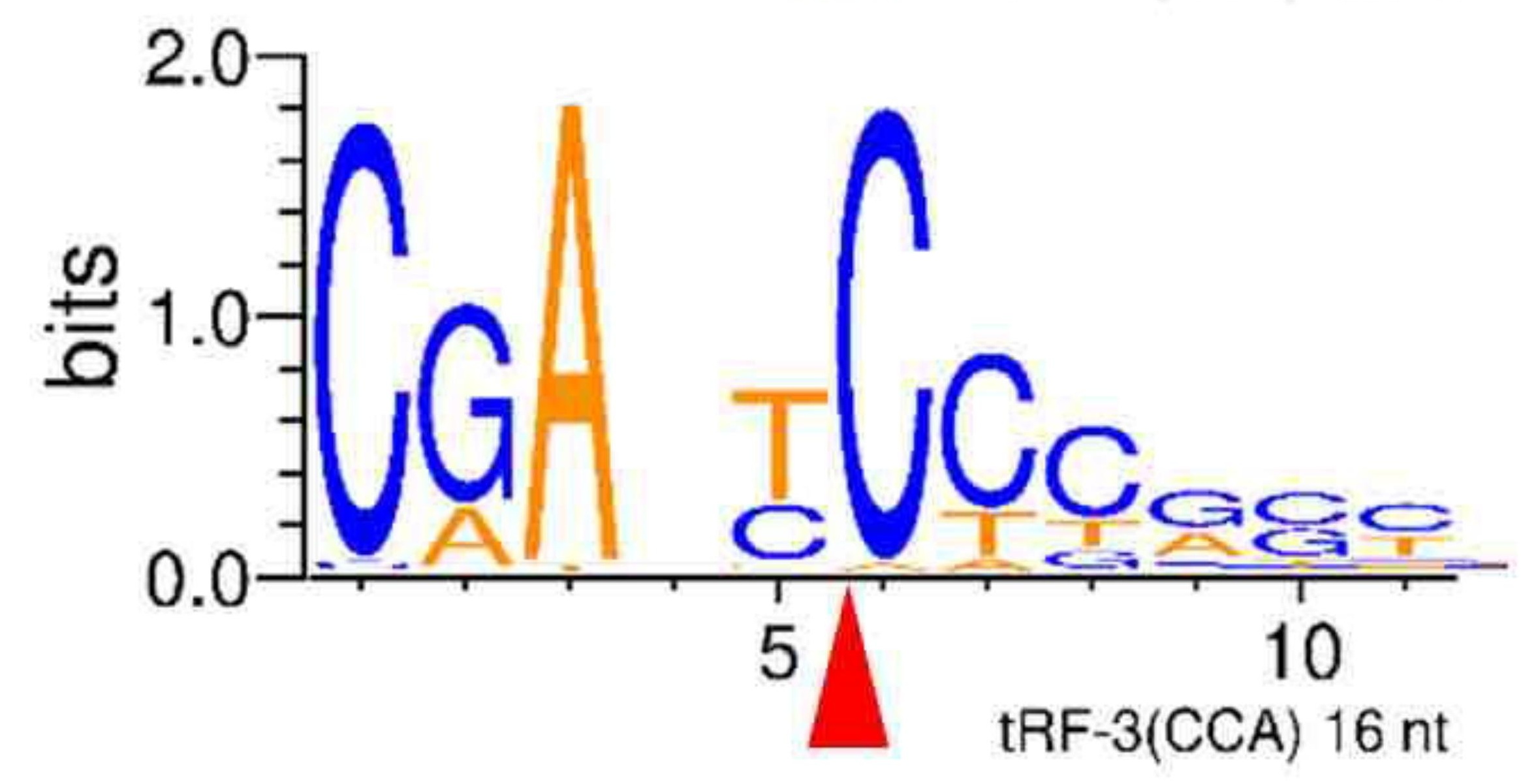
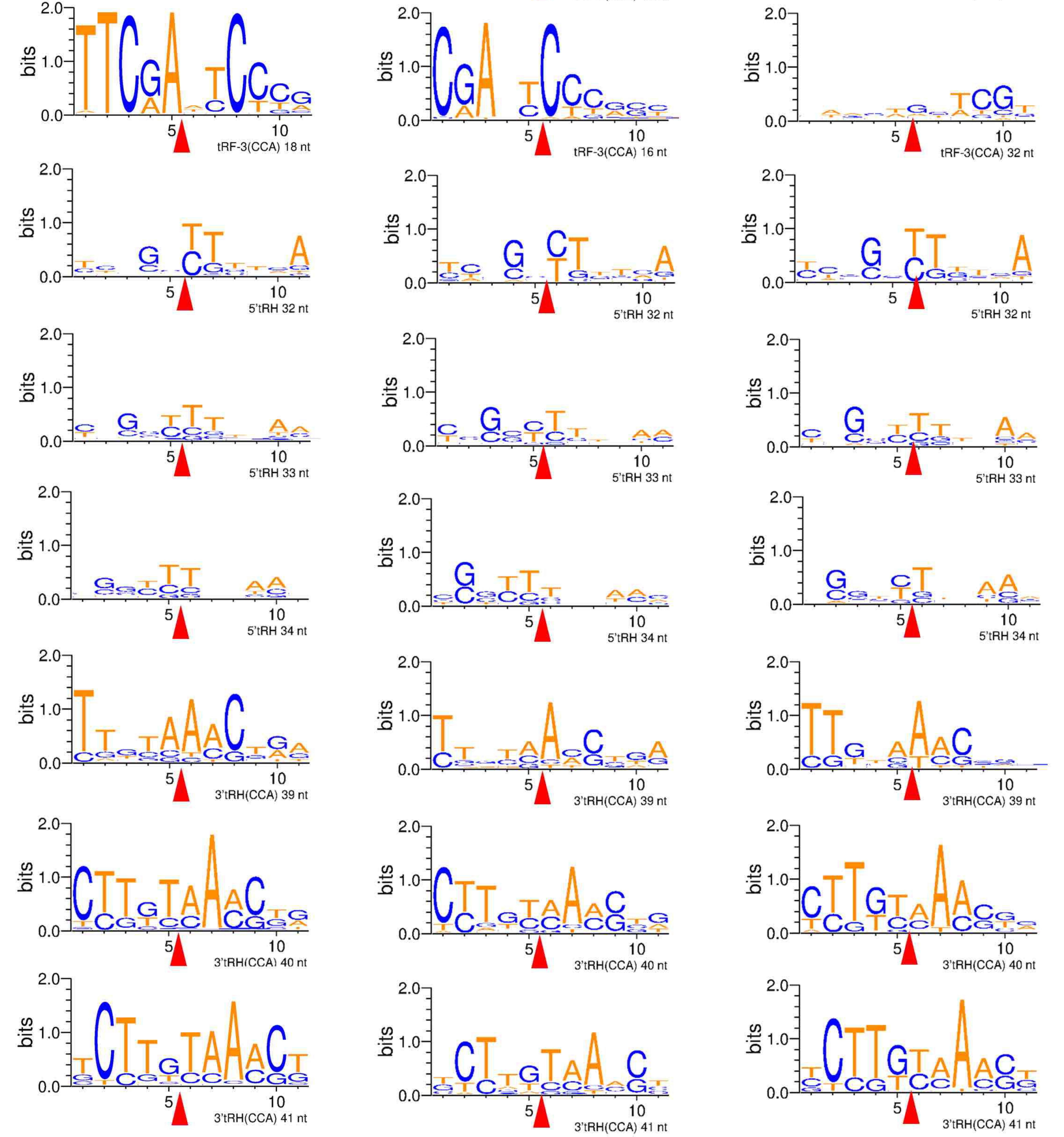

C. arietinum

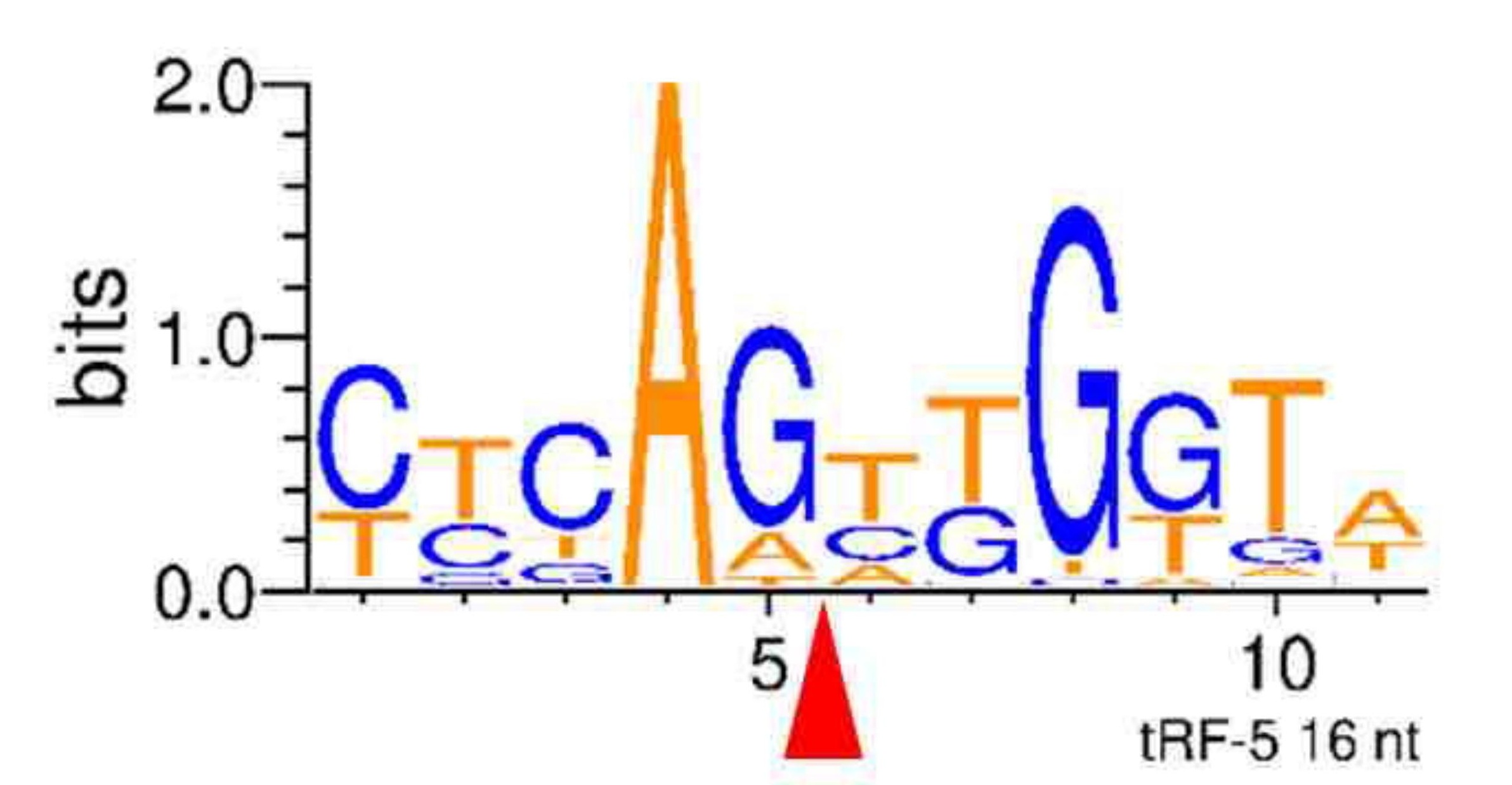

鱼1.0.

鱼1.0
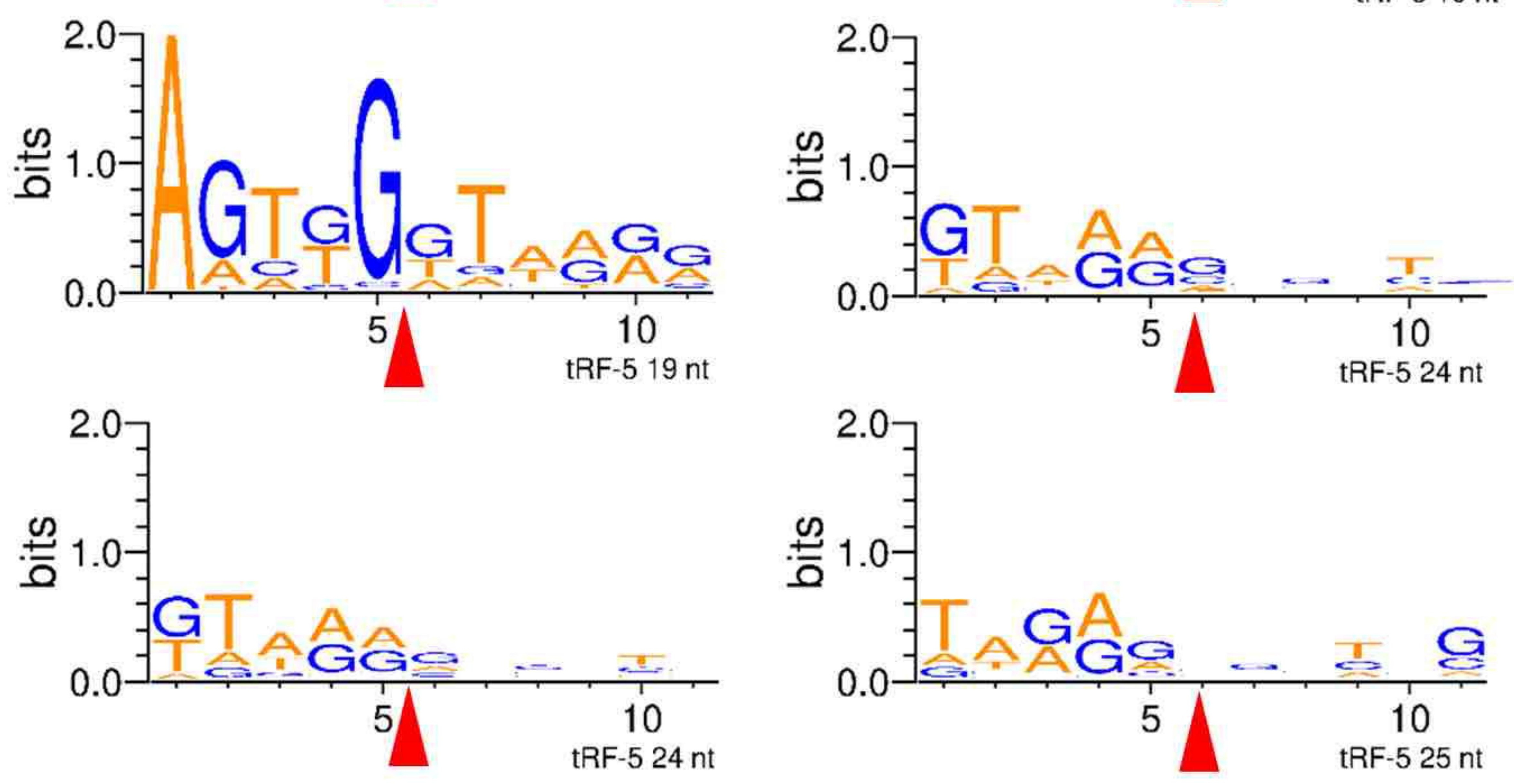

色1.0

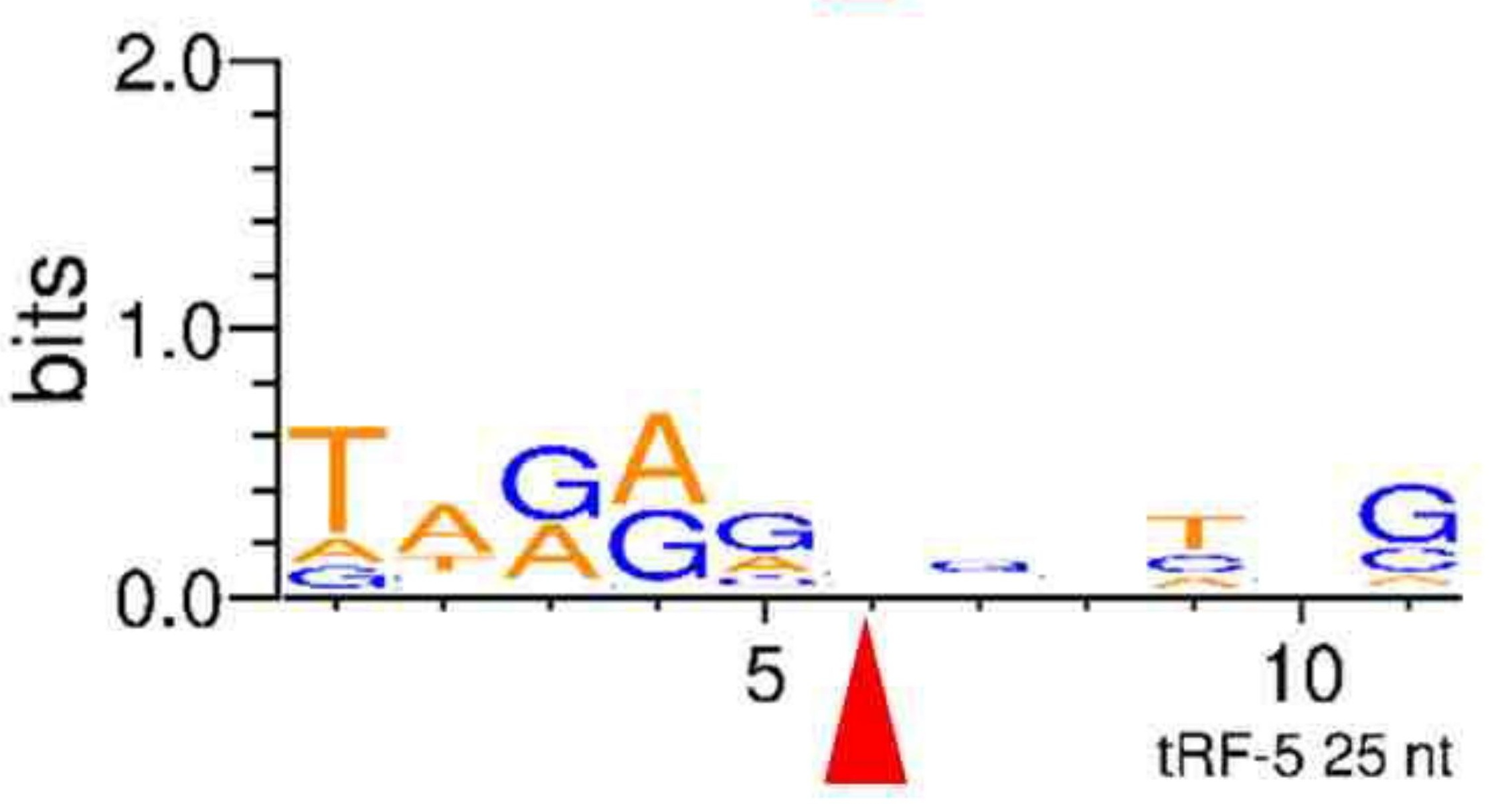

言1.0

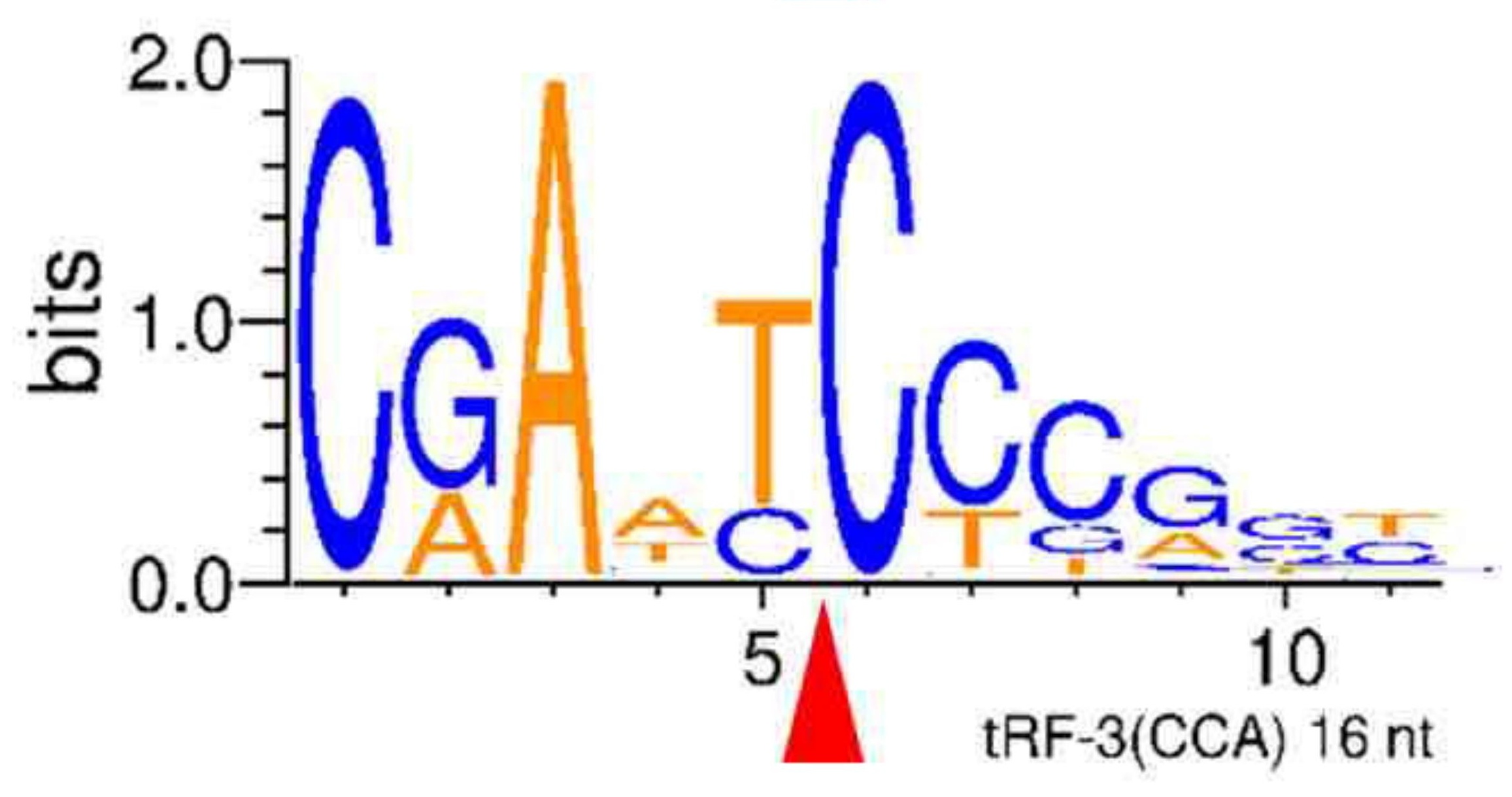


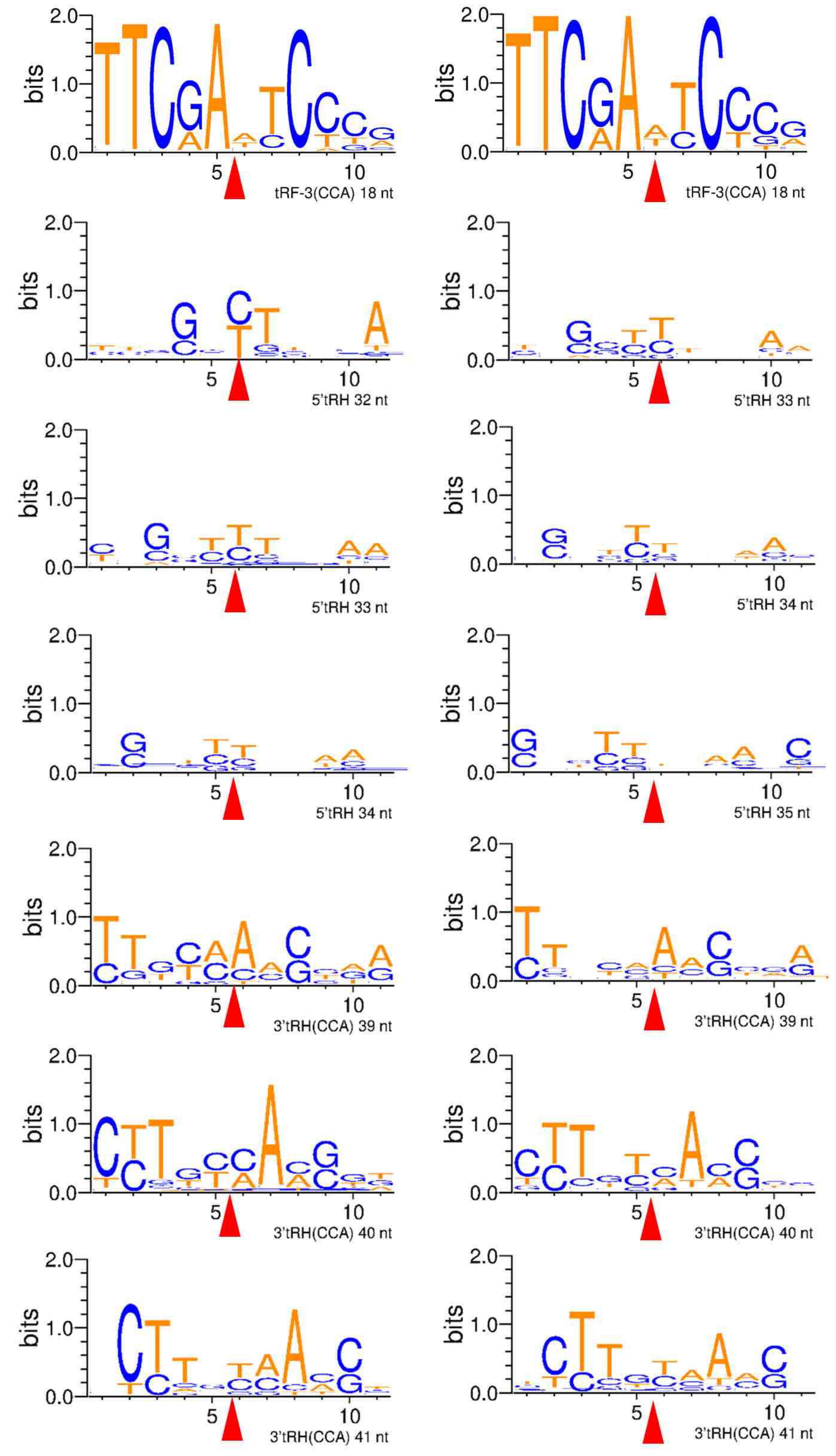

M. truncatula

O. sativa

Z. mays 




A

\section{C}

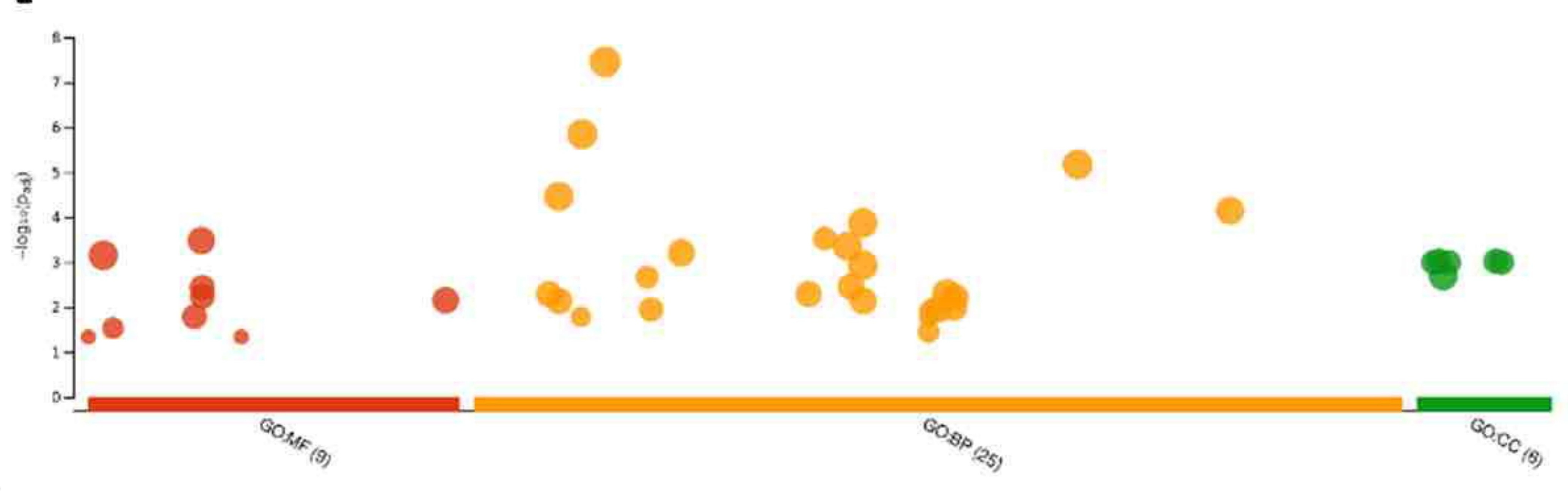

B

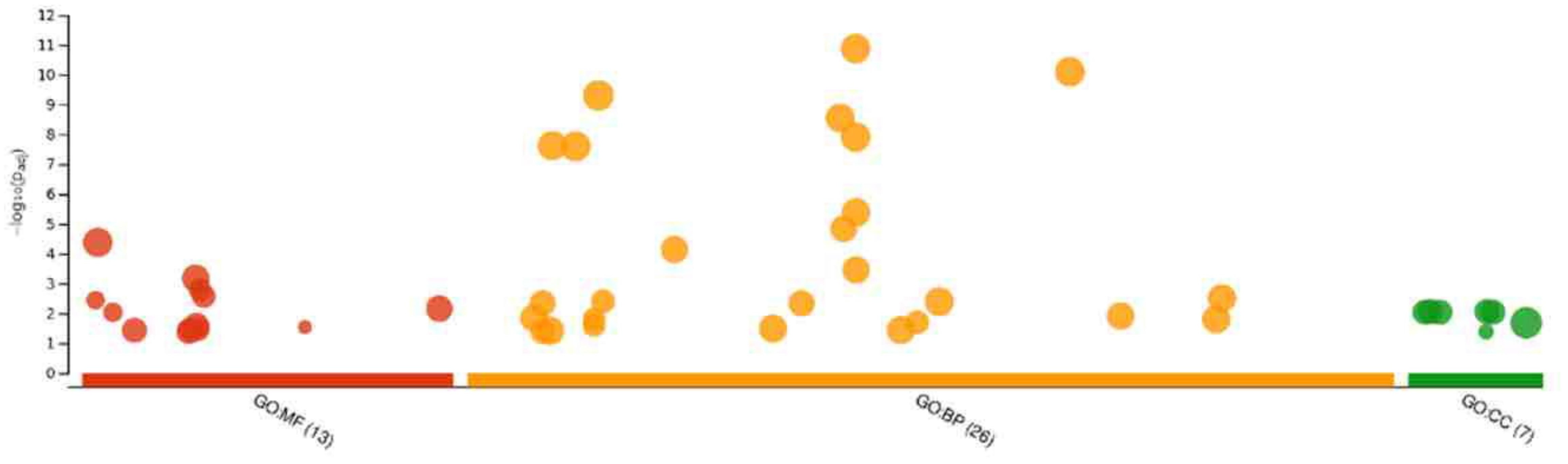

D

\begin{tabular}{|c|c|c|c|c|}
\hline \multicolumn{2}{|l|}{ GO:MF } & \multicolumn{3}{|l|}{ stats } \\
\hline Term name & Term ID & Padj & $0^{-\log _{10}\left(P_{a d}\right)}$ & 26 \\
\hline transferase activity & GO:0016740 & $3.327 \times 10^{-4}$ & & \\
\hline catalytic activity & GO:0003824 & $7.038 \times 10^{-4}$ & & \\
\hline transferase activity, transferring phosphorus-containing groups & G0:0016772 & $3.678 \times 10^{-3}$ & & \\
\hline phosphotransferase activity, alcohol group as acceptor & GO:0016773 & $5.834 \times 10^{3}$ & & \\
\hline catalytic activity, acting on a protein & GO:0140096 & $7.066 \times 10^{-3}$ & & \\
\hline kinase activity & GO:0016301 & $1.643 \times 10^{-2}$ & & \\
\hline endopeptidase activity & GO:0004175 & $2.933 \times 10^{2}$ & & \\
\hline actin-dependent ATPase activity & GO:0030898 & $4.627 \times 10^{-2}$ & F & \\
\hline microfilament motor activity & GO:0000146 & $4.627 \times 10^{-2}$ & F & \\
\hline
\end{tabular}

GO:BP

Term name

cellular process

metabolic process

organic substance metabolic process

nitrogen compound metabolic process

organonitrogen compound metabolic process

primary metabolic process

growth

macromolecule metabolic process

protein metabolic process

cellular metabolic process

cell growth

macromolecule modification

response to stimulus

protein modification process

cellular protein modification process

establishment of localization

cellular protein metabolic process

transport

localization

anatomical structure development

phosphorylation

developmental growth

thythmic process

circadian rinythm

meristem development stats

\begin{tabular}{l|l} 
Term ID & P \\
\hline
\end{tabular}

GO:0009987

60:0008152

GO:0071704

GO:0006807

GO:1901564

GO:0044238

60:0040007

GO:0043170

G0:0019538

GO:0044237

60:0016049

GO:0043412

GO:0050896

G0:0036211

60:0006464

GO:0051234

G0:0044267

G0:0006810

GO:0051179

GO:0048856

G0:0016310

60:0048589

GO:0048511

GO:0007623

60:0048507
$3.499 \times 10^{-8}$

$1.421 \times 10^{-6}$

$6.704 \times 10^{-6}$

$3.430 \times 10^{-5}$

$7.134 \times 10^{5}$

$1.347 \times 10^{-4}$

$2.981 \times 10^{-4}$

$4.387 \times 10^{4}$

$6.229 \times 10^{-4}$

$1.173 \times 10^{-3}$

$2.158 \times 10^{-3}$

$3.512 \times 10^{-3}$

$5.030 \times 10^{-3}$

$5.164 \times 10^{-3}$

$5.164 \times 10^{-3}$

$6.069 \times 10^{-3}$

$7.320 \times 10^{-3}$

$7.334 \times 10^{-3}$

$1.001 \times 10^{-2}$

$1.093 \times 10^{-2}$

$1.131 \times 10^{-2}$

$1.208 \times 10^{-2}$

$1.656 \times 10^{-2}$

$1.656 \times 10^{-2}$

$3.558 \times 10^{-2}$ $0^{-\log _{10}\left(P_{a d}\right)}$

D. Term name

primary metabolic process

organic substance metabolic process

cellular process

macromolecule metabolic process

cellular metabolic process

nitrogen compound metabolic process

metabolic process

cellular macromolecule metabolic process

macromolecule modification

protein metabolic process

cellular protein metabolic process

organonitrogen compound metabolic process

fruit development

response to stimulus

cellular protein modification process

protein modification process

nucleobase-contin

embryo development ending in seed dormancy

organic cyclic compound metabolic process

seed development

embryo development

cellular nitrogen compound metabolic process

heterocycle metabolic process

cellular aromatic compound metabolic process

protein phosphorylation stats

Term ID

GO:0009506

G0:0055044

G0:0005911

GO:0030054

GO:0070161

60:0016020

stats
Padj $\quad 0^{-\log _{10}\left(P_{\text {adj }}\right)}$

$9.571 \times 10^{-4}$

$9.571 \times 10^{-4}$

$1.009 \times 10^{3}$

$1.009 \times 10^{-3}$

$1.009 \times 10^{-3}$

$2.008 \times 10^{-3}$ $0^{-\log _{10}\left(P_{\text {adj }}\right)}+.56$

\begin{tabular}{|l|l|l|l|}
\hline GO:CC & & stats & \\
\hline Term name & Term ID & Padj & $-\log _{10}\left(P_{\text {Padj }}\right)$ \\
\hline symplast & $60: 0055044$ & $8.682 \times 10^{-3}$ & \\
plasmodesma & $60: 0009506$ & $8.682 \times 10^{-3}$ & \\
anchoring junction & $60: 0070161$ & $9.159 \times 10^{-3}$ & \\
cell junction & $60: 0030054$ & $9.159 \times 10^{-3}$ & \\
cell-cell junction & $60: 0005911$ & $9.159 \times 10^{-3}$ & \\
cellular anatomical entity & $60: 0110165$ & $2.070 \times 10^{-2}$ & \\
perinuclear region of cytoplasm & $60: 0048471$ & $4.179 \times 10^{-2}$ & \\
\hline
\end{tabular}

E

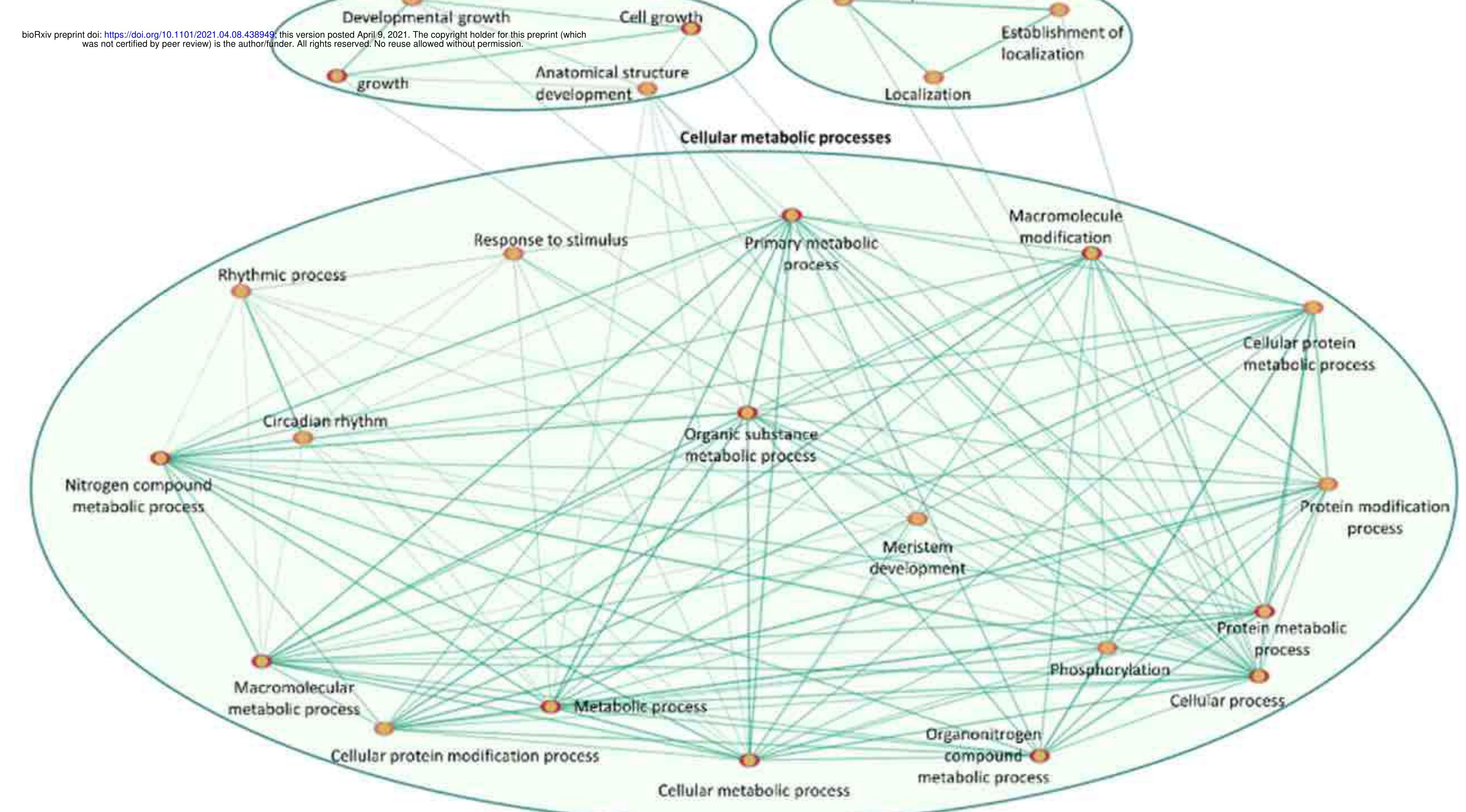

$\mathbf{F}$
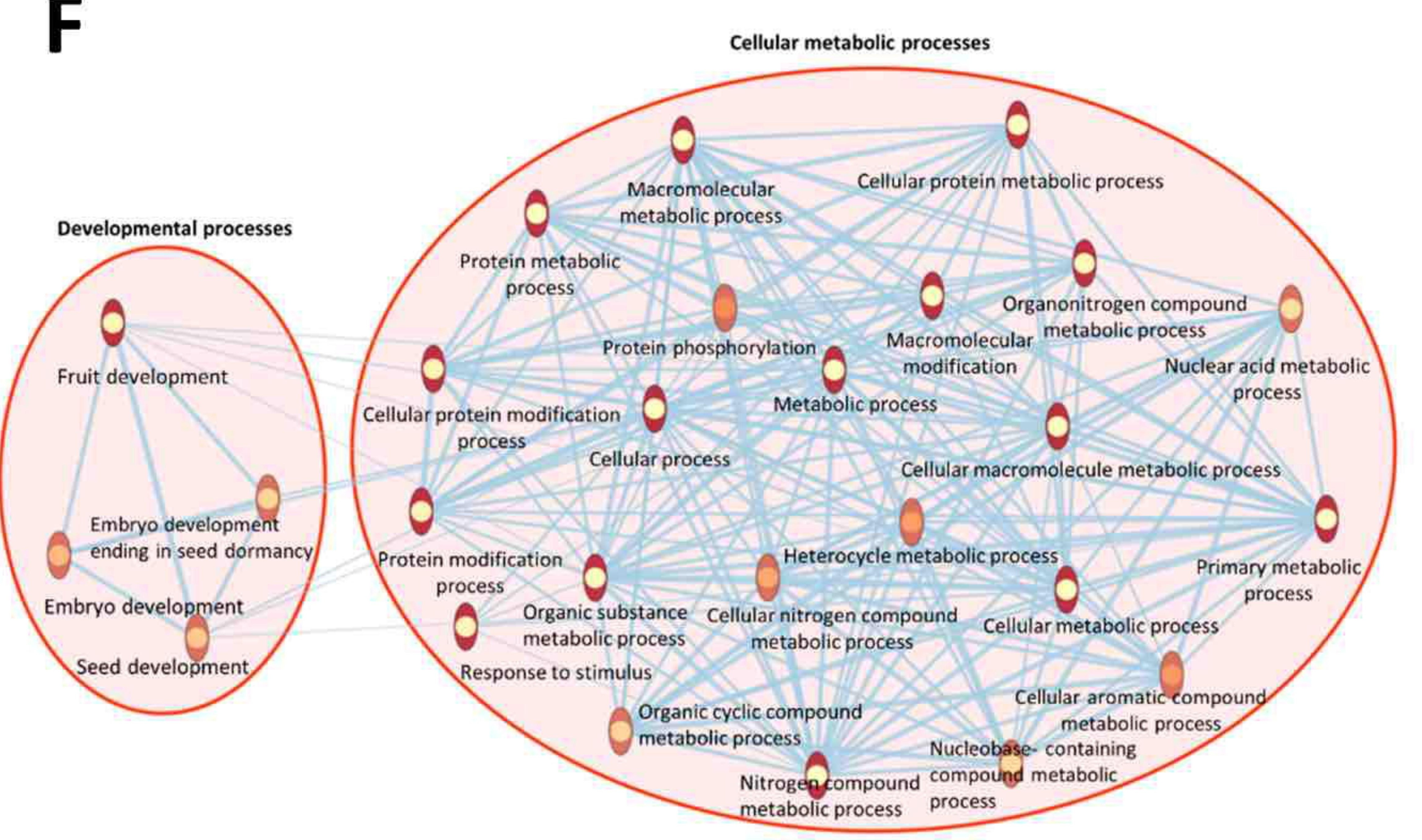

metabolic process process stat:

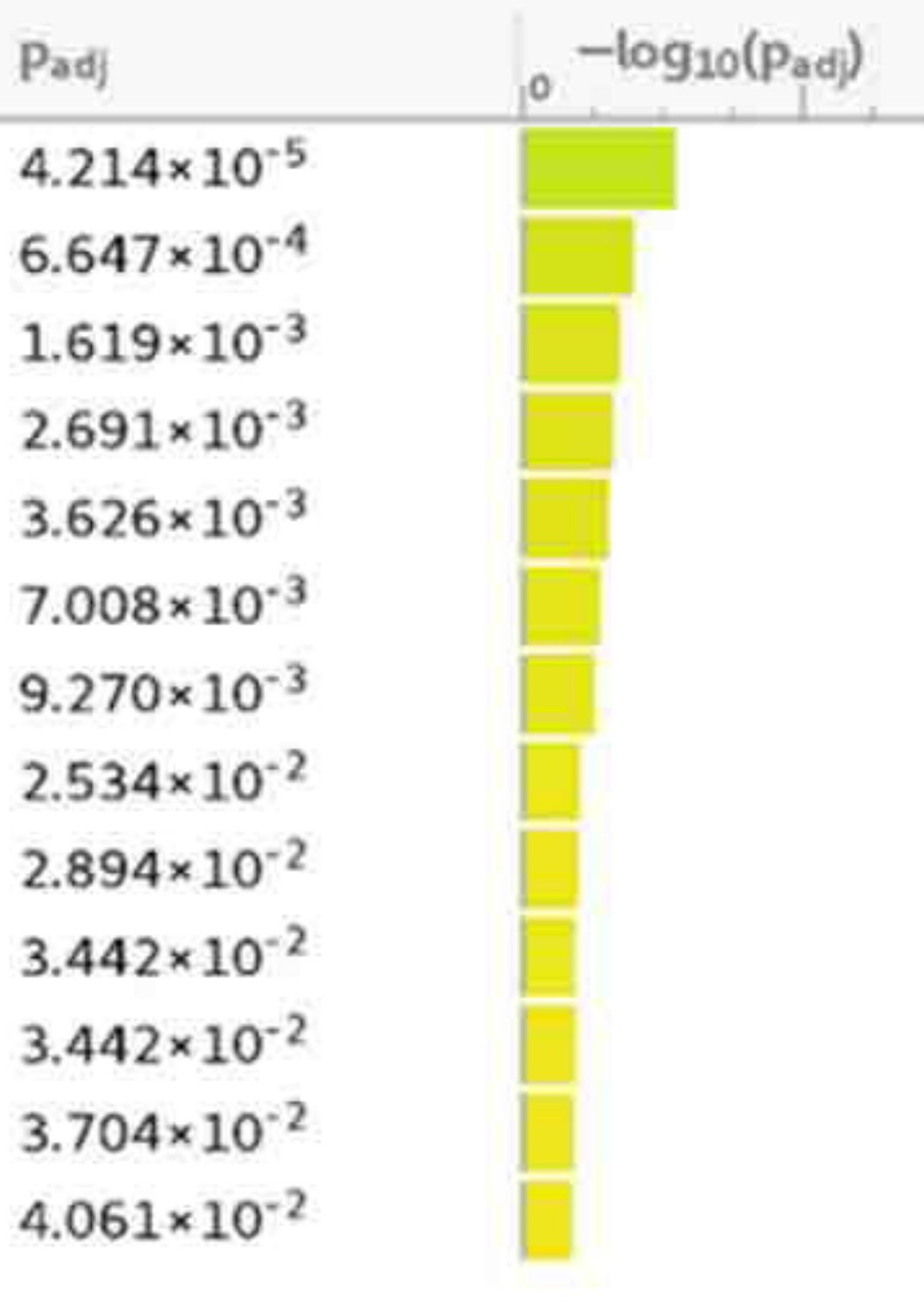

tats

Padj

$1.342 \times 10^{-11}$

$7.956 \times 10^{-11}$

$4.921 \times 10^{-10}$

$2.843 \times 10^{-9}$

$1.226 \times 10^{-8}$

$2.386 \times 10^{-8}$

$2.539 \times 10^{-8}$

$1.476 \times 10^{-5}$

$7.242 \times 10^{-5}$

$3.534 \times 10^{-4}$

$3.092 \times 10^{-3}$

$3.920 \times 10^{-3}$

$4.628 \times 10^{-3}$

$4.628 \times 10^{-3}$

$1.232 \times 10^{-2}$

$1.427 \times 10^{-2}$

$1.561 \times 10^{-2}$

$1.585 \times 10^{-2}$

$2.004 \times 10^{-2}$

$2.502 \times 10^{-2}$

$3.232 \times 10^{-2}$

$4.200 \times 10^{-2}$

membrane 


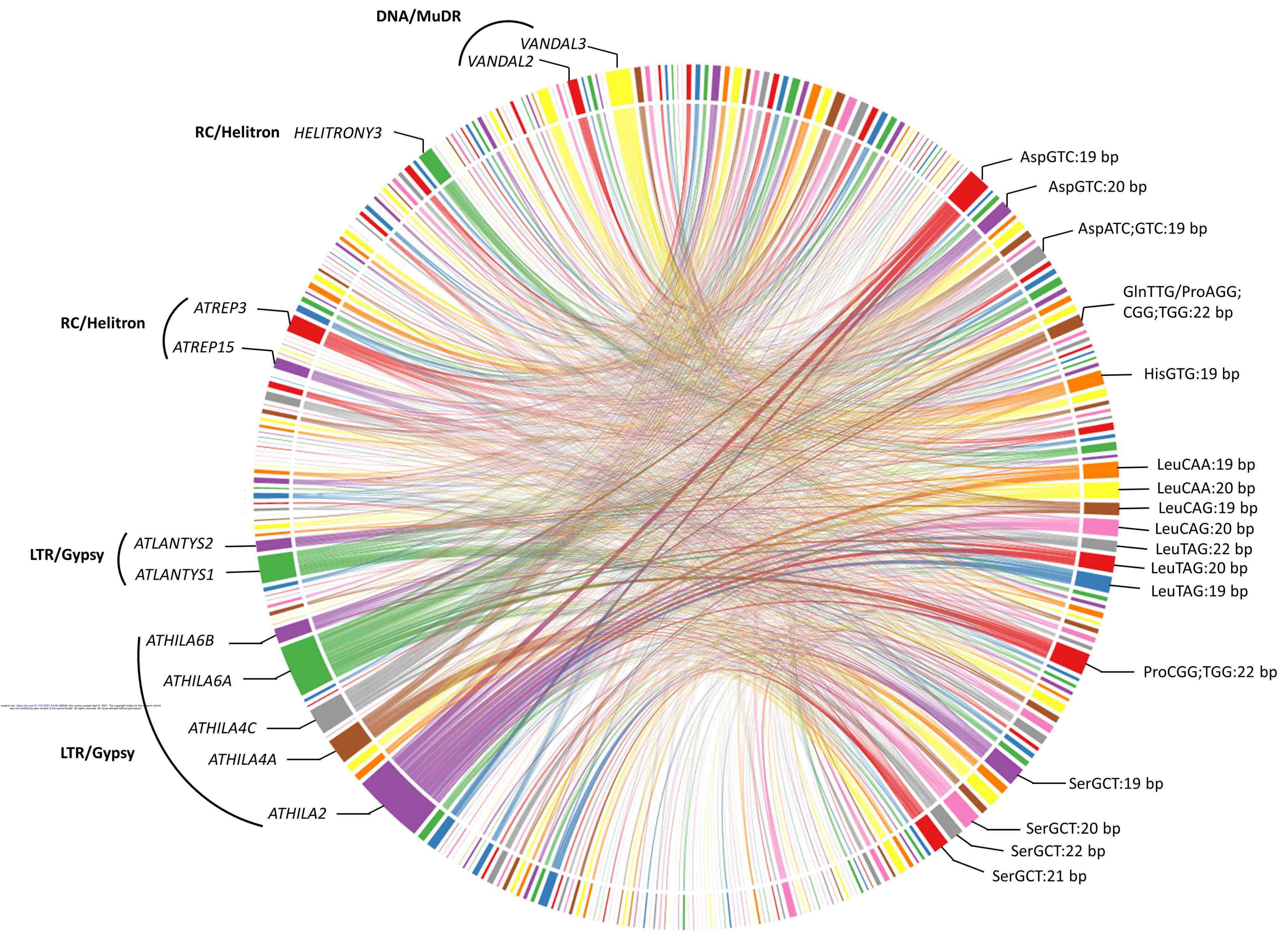



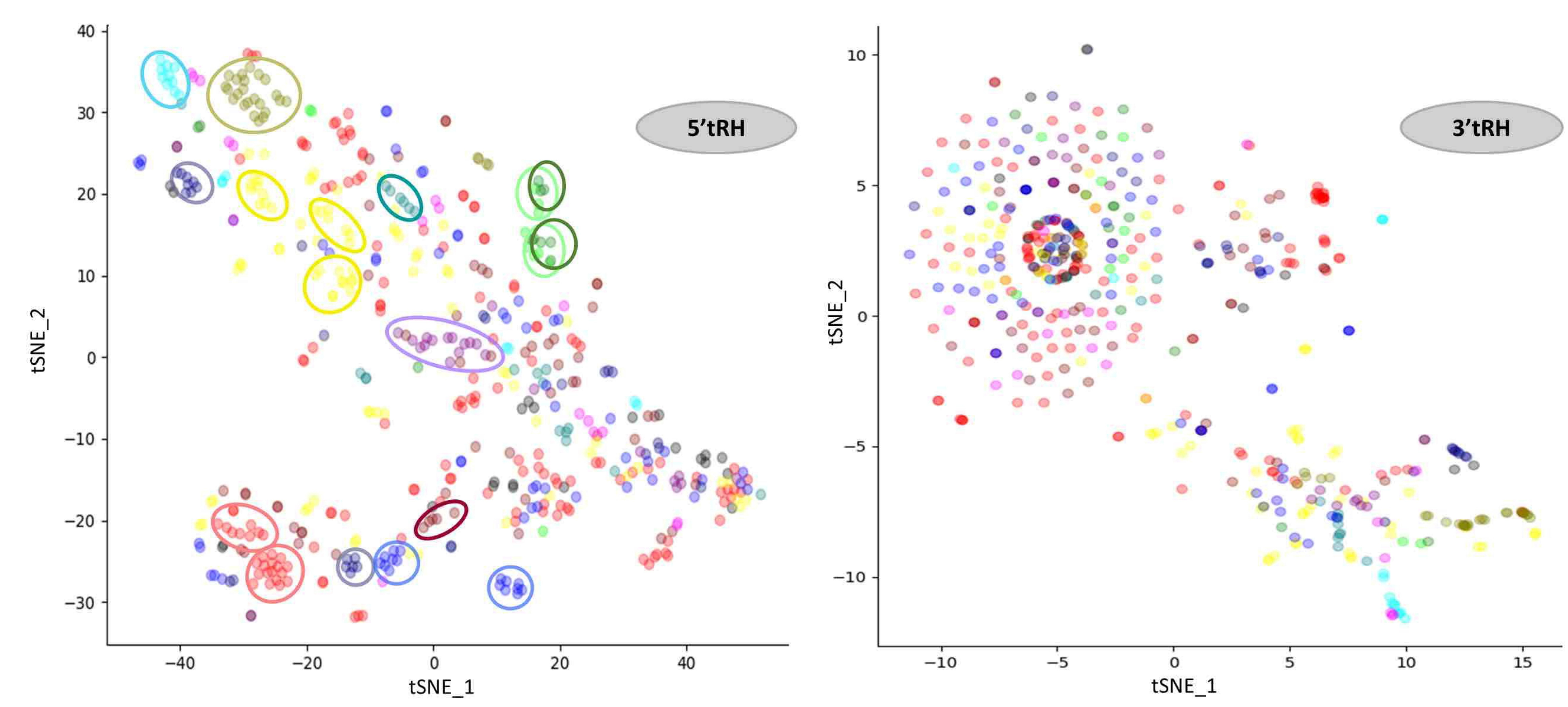

Rosette leaf

Seedling

- Leaf

Inflorescence

Seed

Vasculature

Epidermis

Whole plant

Pollen

Flower

Shoot

Root

Silique
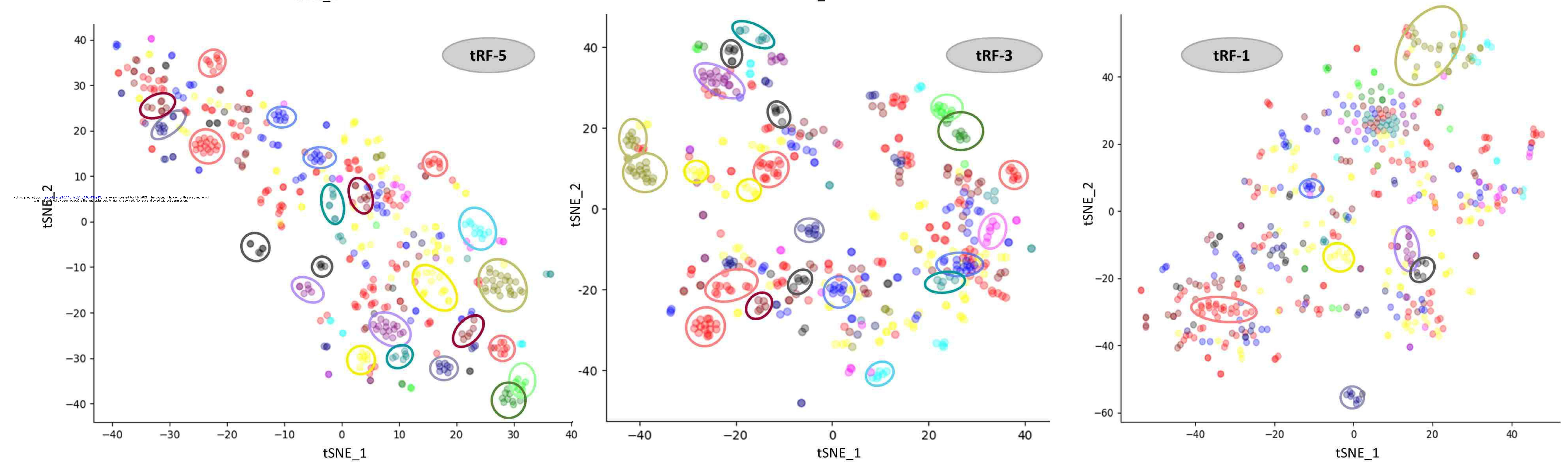

